Ethiopian Journal of Environmental Studies \& Management 9(6): 713 - 737, 2016.

ISSN:1998-0507

Submitted: June 17, 2016

doi: http://dx.doi.org/10.4314/ejesm.v9i6.5

\title{
EVALUATION AND FLORA DIVERSITY OF GASHAKA GUMTI NATIONAL PARK-1, GASHAKA SECTOR, TARABA STATE, NIGERIA
}

\author{
AKINSOJI, A., ${ }^{1}$ ADEONIPEKUN, P.A., ${ }^{1}$ *ADENIYI, T.A., ${ }^{1,2}$ OYEBANJI, 0.0. ${ }^{1}$ AND \\ ELUWOLE, T.A. ${ }^{2}$ \\ ${ }^{1}$ Department of Botany, Faculty of Science, University of Lagos, Akoka, Lagos, Nigeria \\ ${ }^{2}$ Environmental Accord Nigeria Limited, Gbagada, Lagos, Nigeria
}

\begin{abstract}
The alarming rate of ecosystem degradation in many parts of Nigeria has continued to have significant impacts on the country's resources, conservation potential, and climate. This pressure led to an ecological and geographical survey in Gashaka Gumti National Park 1, Taraba State, Nigeria in 2013 and 2015 to document the species composition across the different vegetation types and geomorphic gradients, and to assess the vegetation loss between 1991 and 2013. Vegetation analysis was done using standard ecological sampling procedures. Digital Elevation Mapping (DEM) was also done to estimate the altitudinal ranges of the Park, while a Normalized Difference Vegetation Index (NDVI) was calculated to ascertain vegetation loss over time. Recognized vegetation types are lowland rainforest, southern Guinea savanna, and montane. A total of 426 species were identified belonging to 306 genera and 104 families with Asteraceae dominating (37 spp.). Also, five species which have never been formally documented in any published flora of Nigeria were identified. With respect to habit, 29 grasses, 26 ferns, 19 climbers, 98 forbs, 9 sedges, 79 shrubs, 152 trees and 14 epiphytes were classified. Plant diversity decreased with increasing altitude. The DEM classified the heights of the sampled area into lowland $(400-933 \mathrm{~m})$, sub-montane $(933-1467 \mathrm{~m})$, and montane $(1467-2000 \mathrm{~m})$. The NDVI revealed higher vegetation cover in 1991 than 2013. These results indicate the importance of regular assessment of floristic composition through checklisting of species in national parks for effective and efficient species conservation and management.
\end{abstract}

Key Words: Gashaka Gumti, National Park, floral diversity, geomorphic gradient, DEM and NDVI

\section{Introduction}

The Nigeria vegetation is one of the most endowed in Africa containing almost all the vegetation types that exist in other African countries widely distributed in different zones of the country. Nigeria harbors about 7895 species of plant; and this makes it one of the richest countries in the continent in terms of biodiversity (Ayodele and Yang, 
2012). However, ecosystem degradation proceeds at alarming rates in many parts of Nigeria, including some protected areas which are meant to be conserved (Gumnior and Sommer, 2012). Ladipo (2010) indicated that nearly $90 \%$ of rainforests in Nigeria has been cleared as at 2006. This clearance is not restricted to the forests alone as all habitats are under threat from civilization and other unsustainable human activities. The careless attitude of the populace and high rate of poverty in the country has also resulted in this heavy loss (Ayodele and Yang, 2012).

In order to prevent forest loss and to preserve biodiversity, the Nigerian Government has established several national parks. One of the national parks established by Government is Gashaka Gumti National Park (GGNP). It is the largest National Park in Nigeria covering an area of $6402 \mathrm{~km}^{2}$, lies between $6^{\circ}-8^{\circ}$ latitude and $11^{\circ}-12^{\circ}$ longitude (Akinsoji, 2003). GGNP is divided into the southern Gashaka Sector which is situated in Taraba State and the Northern Gumti sector situated in Adamawa State. The Gumti sector has an undulating topography covered by Savanna vegetation while the topography of Gashaka sector is rough, rugged and mountainous (Akinsoji, 2003). Rivers originate from the mountain tops and flow down the mountain thus providing moisture which sustains the lowland forest in addition to the savanna vegetation at lower altitudes. This sector therefore has a variety of vegetation types including lowland rainforest, woodland savanna, montane forest and montane grassland.

These vegetation types provide variety of habitats and microhabitats for plants and animals. Habitat diversity promotes biodiversity thus accounting for the high diversity of GGNP (Oates et al., 2014). The pioneering ecological study of the park was documented in Akinsoji (1994, 1996), which were unpublished preliminary vegetation surveys done as part of the Nigerian Concervation Foundation - WWF - UK Conservation Project. The first published vegetation study of the GGNP addressed specifically the ethnobotany of the park to show how the inhabitants have been using these plants for their survival (Akinsoji, 2003). Akinsoji et al. (2003) relied on the natural state of the vegetation of this park to compare the Point-Centered Quarter and Quadrant methods to analyze the phytosociology of its trees. Akinsoji (2005) also carried out a survey of epiphytes of the GGNP and reported that the epiphytic flora was dominated by orchids and these orchids preferred Entada sp. as host plant. Others works include Ayodele and Yang (2012) and Gumnior and Sommer (2012). From the analysis of species density and diversity along geomorphic gradient, Mubi and Tukur (2012) also reported some vegetation studies in GGNP. They noted that the plains and riparian areas within the GGNP had the highest mean density and diversity. Most recently anthropogenic activities such as illegal grazing, farming, and poaching have caused a concern for conservation particularly grazing, where the herdsmen have not only grazed in the park but attacked Park staff when confronted. Dunn (1995) had earlier reported increasing pressure on GGNP as a result of its situation within the sub-humid zone, and its exposure to immigration impacts from more densely cultivated 
northern and southern sections. Also, revelation from satellite imagery, shows that during most of the dry season, green vegetation was absent in up to one fifth of the park (Gumnior and Sommer 2000). This was attributed largely to human activities such as cattle grazing and burning. Gumnior and Sommer (2012) further confirmed environmental degradation detected in previous imagery, and established a trend of degradation in the newer scenes.

This degradation has necessitated the need for effective and holistic conservation and management policy that requires among others efforts, a comprehensive data gathering on its floral and fauna diversity. Therefore, this present effort documents species composition and conservation status, as well as evaluates the impact of anthropogenic activities on the park using both conventional vegetation study and GIS mapping.

\section{Materials and Methods Study Area}

The area under study is a subset of GGNP that lies between $07^{\circ} 18^{\prime} \mathrm{E}$ and $07^{\circ} 24^{\prime} \mathrm{N}$ and $11^{\circ} 34^{\prime} \mathrm{E}$ and $11^{\circ} 46^{\prime} \mathrm{E}$ with an area of about $289 \mathrm{~km}^{2}$ situated within Gashaka LGA (Fig. 1). The park has a multitude of crucial ecological functions by encompassing most of the catchment of the Taraba River, largest tributary to river Benue. It is divided into the undulating Gumti sector in the north and the hilly to mountainous Gashaka sector in the south, where elevations rise to 2,419 $\mathrm{m}$ asl (above sea level) at Gangirwal, Nigeria's highest peak (Gumnior and Sommer, 2012). Located in the tropical wet and dry climatic zone, Kwano field station (583 $\mathrm{m}$ asl, $7^{\circ} 19^{\prime} \mathrm{N}$, $11^{\circ} 35^{\prime}$ E) has an average total annual precipitation of $1,973 \mathrm{~mm}$ (period: 2001 2002/2004-2008). About $95 \%$ of the yearly rainfall is recorded between April and October, with highest monthly mean in September $(332 \mathrm{~mm})$. The influence of Sahara Trade Winds (Harmattan / N/East Trade winds) accounts for the absence of rainfall between December and January (Sommer and Ross, 2011). Significantly higher precipitation falls along the southeastern escarpments and allows lush rainforests to thrive albeit southern Guinea savanna is considered to be the zonal climax (Keay, 1959). The steep terrain is also responsible for the occurrence of sub-montane and montane vegetation types, contributing to a highly complex forest-savanna mosaic (Keay, 1959). Deforestation and dry season burning are believed to have turned considerable parts of semi-deciduous forests into woodlands and led to the prevalence of extensive grasslands at the expense of montane forests (Chapman et al., 2004). Since its creation, GGNP has been harboring several settlements and grazing enclaves, with an estimated overall human population of 5,000 and about 10,000 cattle. Thus, legal and illegal human activities within the park such as grazing, burning, cutting of trees, and poaching, together with the poisoning of carnivores to protect cattle, have always remained an issue, sometimes resulting in violent conflicts with National Park staff. This does not only create numerous conservation challenges regarding wildlife, but puts increasing pressure on all natural resources especially in the vicinity of the enclaves (Chapman et al., 2004; Sommer and Ross, 2011). 


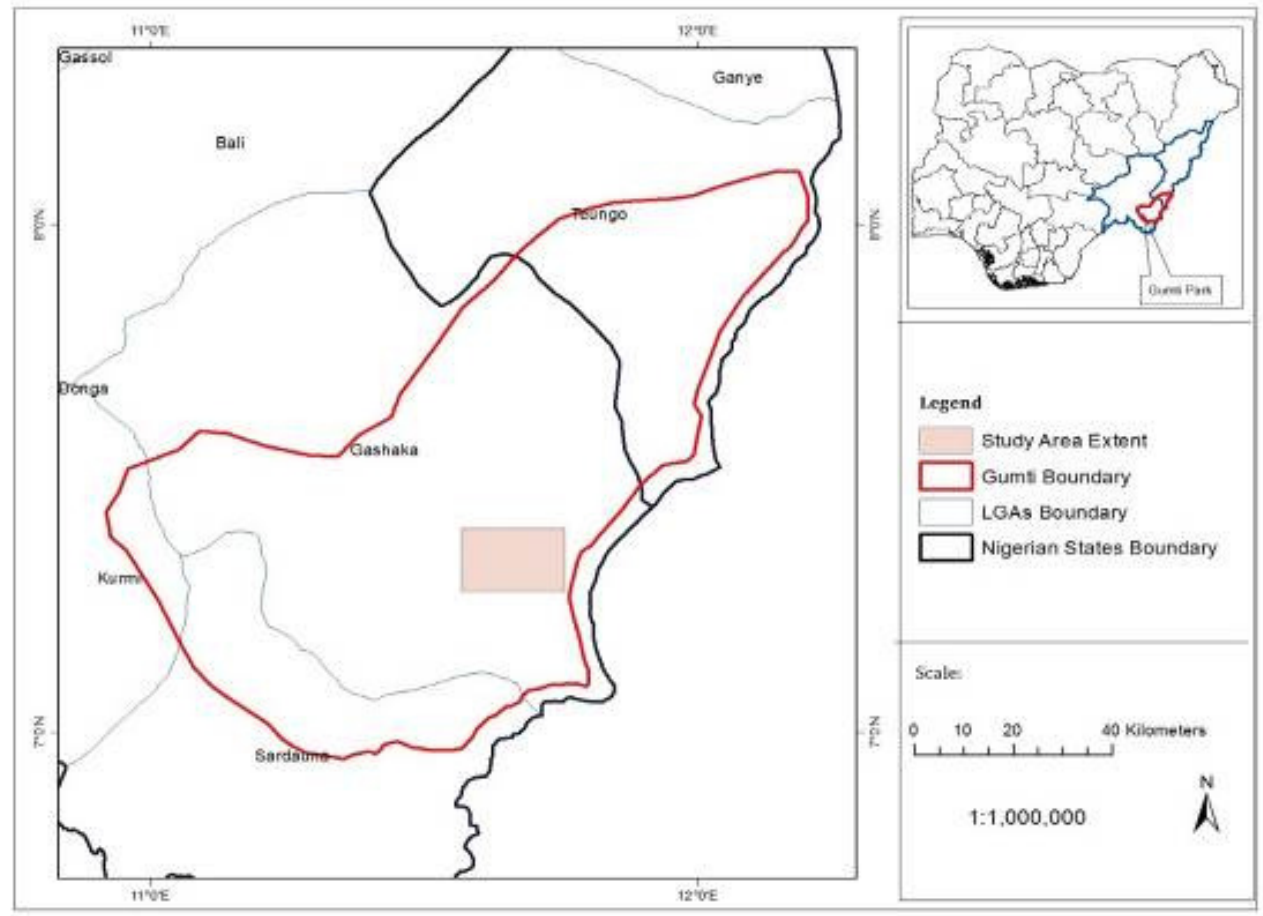

Figure 1: Map of Gashaka Gumti National Park showing study area

\section{Vegetation Study}

A reconnaissance study was first carried out in August 2013 while a formal vegetation study was done in October 2015. Notable information on the vegetation description and species checklist from Gidan kwano (500 m asl) to Chabbal hendu (1,980 $\mathrm{m}$ asl) during the reconnaisance. The ranges consist of lowland ( $<1000 \mathrm{~m}$ asl) with Kwano and Gashaka as landmarks; sub-montane (1000 - $1500 \mathrm{~m}$ asl) with Tonga and Bale (abandoned communities that have allowed the vegetation to grow) as landmarks; and montane (>1500 m asl) with Selbe, Chabbal Hendu as landmarks.

Lowland: The vegetation is wooded grassland but since the area is traversed by rivers and streams, there is forest vegetation along the water bodies. Altitudinal zone ranged between $500<$ and $1000 \mathrm{~m}$ asl. Sampling was done around Gashaka village (583 $\mathrm{m}$ asl) and Kwano (530 m asl). Sub-montane: The vegetation is mainly woodland savanna but the trees are smaller than those at lower altitude. The altitudinal zone ranged between 1000 and $1500 \mathrm{~m}$ asl and sampling was carried out around abandoned communities such as Tonga andBale (very close to Selbe settlement). However, some Fulani herdsmen still live in some parts purposely to farm and graze. Montane: This zone comprises of grassland on the mountain top and montane forest in the river/streams valleys. The altitude is above $1500 \mathrm{~m}$ asl. The sampling was carried out around at Chabbal Hendu (>1800 m asl). Details of the characteristic species of each geomorphic gradient are contained in Table 1. 
Table 1: Details of the sampled points across the study area

\begin{tabular}{lll}
\hline Coordinates & Elevation & Region \\
\hline $7.32708 ; 11.57879$ & $524.3 \mathrm{~m}$ & Lowland \\
$7.32739 ; 11.58408$ & $560 \mathrm{~m}$ & Lowland \\
$7.35736 ; 11.60246$ & $1240 \mathrm{~m}$ & Sub-montane \\
$7.35300 ; 11.60329$ & $1308 \mathrm{~m}$ & Sub-montane \\
$7.35162 ; 11.61328$ & $1450 \mathrm{~m}$ & Sub-montane \\
$7.35200 ; 11.61525$ & $1500 \mathrm{~m}$ & Sub-montane \\
$7.35342 ; 11.62450$ & $1588 \mathrm{~m}$ & Montane \\
$7.35803 ; 11.73120$ & $1748 \mathrm{~m}$ & Montane \\
$7.35727 ; 11.73060$ & $1755 \mathrm{~m}$ & Montane \\
$7.34741 ; 11.70140$ & $1907 \mathrm{~m}$ & Montane \\
\hline
\end{tabular}

\section{Species Identification}

Identification of all the plants, species collection, identification and photography were carried out along the geomorphic gradient on ten sampling points (Fig. 1 and Table 1) using transects (1 m x $10 \mathrm{~m})$ and quadrats $(10 \mathrm{~m} \times 10 \mathrm{~m})$. All plant specimens encountered during the reconnaissance study were identified to species level either in the field or herbarium, using appropriate floras, manuals and monographs such as Hutchinson and Dalziel (1954, 1958, 1963, 1968, and 1972), Alston (1959), Keay et al. (1964), Lowe and Stanfield (1974), and Akobundu and Agyakwa (1998). Identification was further confirmed at the Forestry Herbarium Ibadan (FHI) Ibadan, and the Lagos University Herbarium (LUH), University of Lagos Akoka, Lagos, Nigeria. Classification into families was based on APG III (2009) and Ayodele and Yang (2012). Representative samples of species were collected, pressed, dried and prepared as herbarium specimens using standard techniques (Radford et al., 1974) and deposited at the Lagos University Herbarium (LUH) University of Lagos, Nigeria.

\section{GIS Analysis}

ASTER DEM data for the study area was obtained from Global Land Facility Cover and imported into the ArcMap 10.0 GIS environment. The contour and slope of the study area were generated sequentially to serve as input raster data for the DEM. The DEM generated was later re-classified into three and overlaid by the sampling points. For Normalized Difference Vegetation Index (NDVI) analysis, maps of GGNP were obtained from LandSat images database. The maps used were images of GGNP taken in the years 1991 and 2013.

\section{Results}

A total of 426 plant species were identified (Plate 1) belonging to 306 genera and 104 families with Asteraceae being the most represented family having 37 species (Table 2). In terms of habit, 29 grasses, 26 ferns, 19 climbers, 98 forbs, 9 sedges, 79 shrubs, 152 trees and 14 epiphytes were classified (Table 2). An inverse relationship was observed between plant diversity and altitude. Plant diversity reduced with increase in altitude. The low land region (Plate 2) had 211 species in 74 families, submontane region 174 species in 54 
families, and montane 147 species in 65 families (Figs. 4 \& 5). Epiphytes (9), grasses (20), and trees (96) were most abundant in the low-land region while ferns (15) and sedges (5) dominate the montane region. The sub-montane had the most abundant climbers (11), forbs (50), and shrubs (46) (Fig. 5).
The DEM classified the heights into three geomorphic gradient classes: lowland $(400-933 \mathrm{~m})$, sub-montane $(933$ - $1467 \mathrm{~m})$, and montane $(1467$ $2000 \mathrm{~m}$ ) (Fig. 2). The mean indices of Band 1 for the LandSat TM images were higher in images from 1991 (0.21) than images from 2013 (0.14) (Figs. 3a and b).

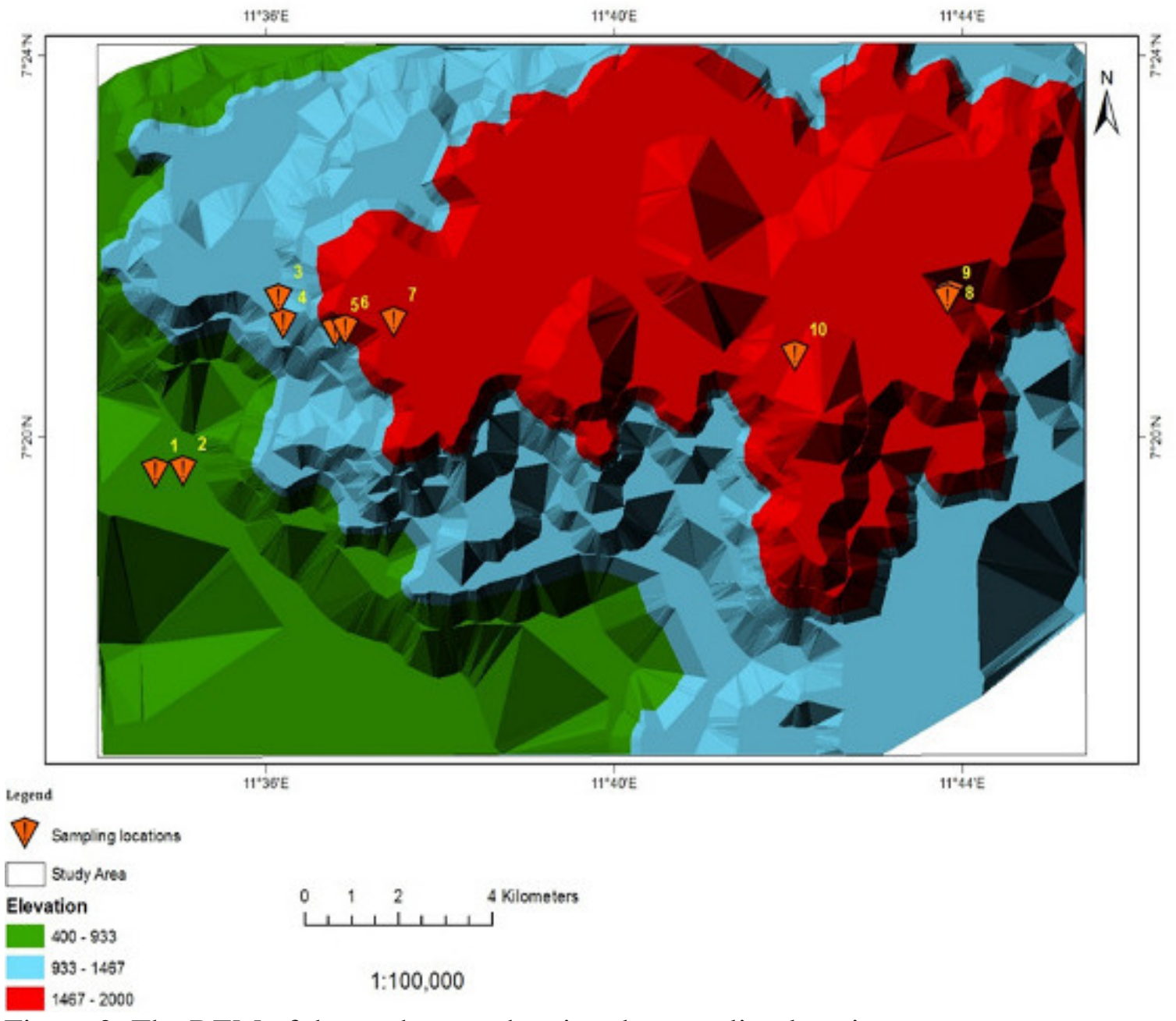

Figure 2: The DEM of the study area showing the sampling locations 
Ethiopian Journal of Environmental Studies and Management Vol. 9 no.6 2016

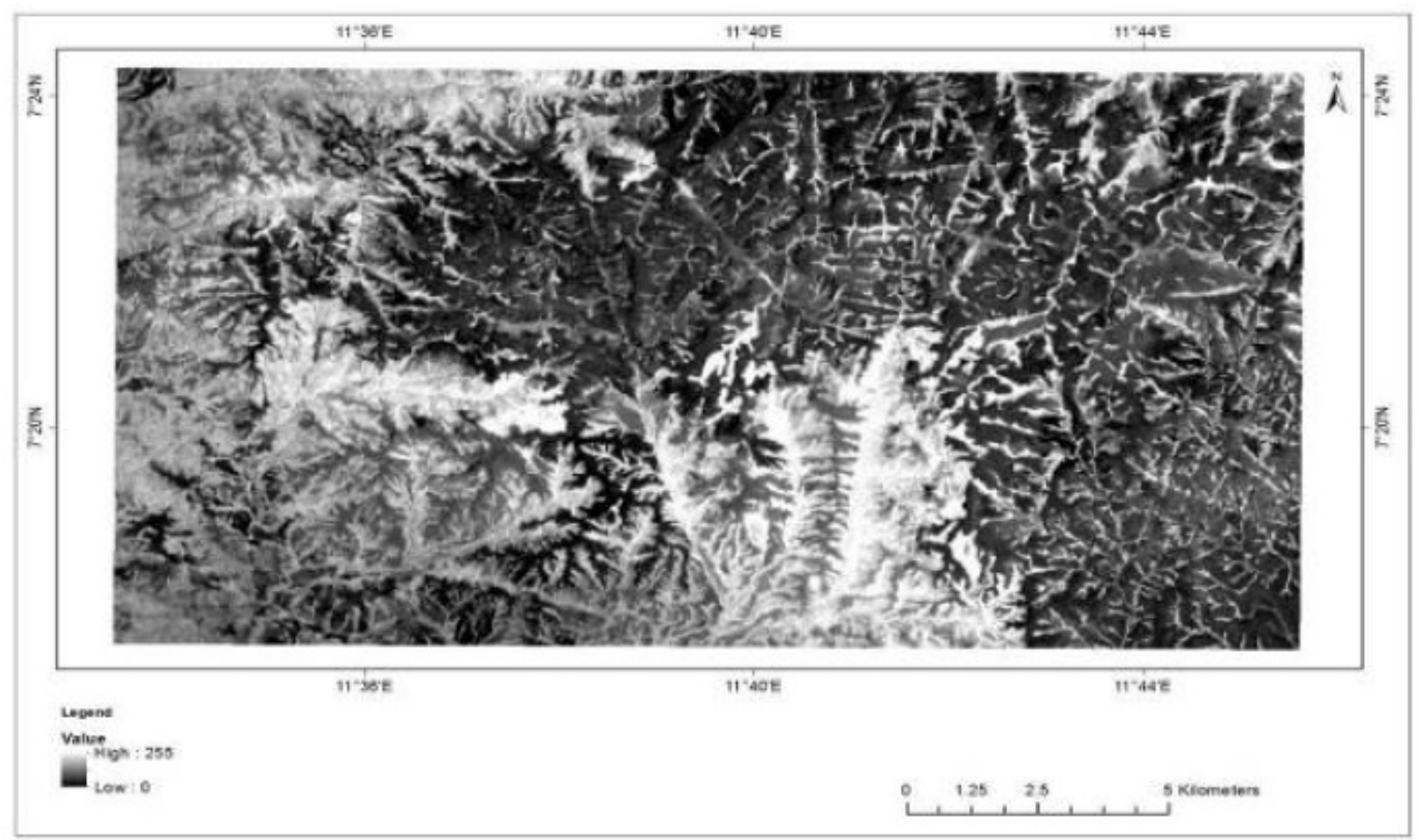

Figure 3a: Normalised Difference Vegetation Index Imagery of GGNP, 1991

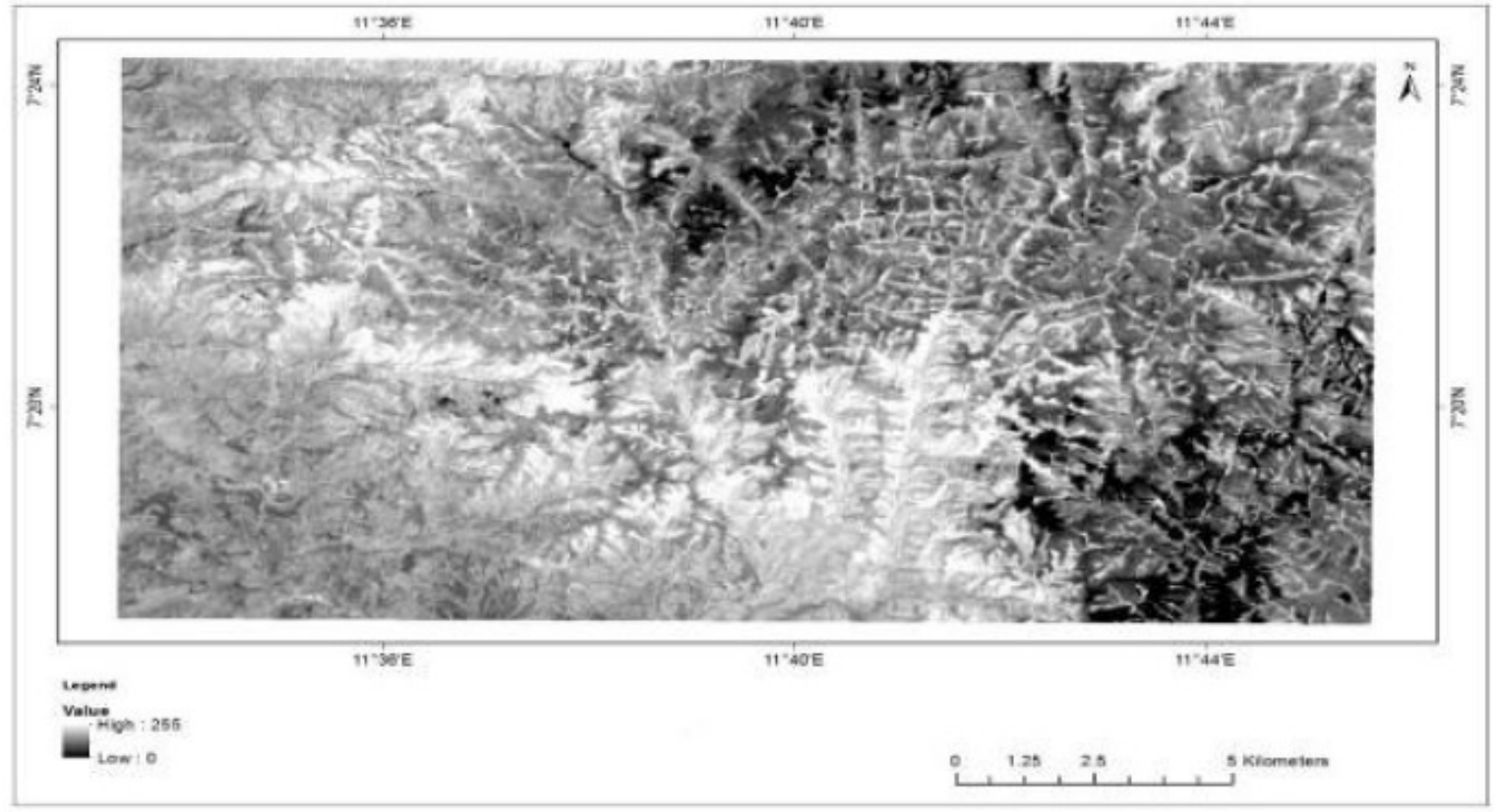

Figure 3b: Normalised Difference Vegetation Index Imagery of GGNP, 2013 
Table 2: Details of Species diversity of GGNP -1

\begin{tabular}{|c|c|c|c|c|c|c|}
\hline Botanical Name & Family & Habit & Low-land & Sub-montane & Montane & IUCN Status \\
\hline Abrus precatorius Linn. & Papilionoideae & Climber & + & & & Not Assessed \\
\hline Acalypha ornata Hochst. ex A. Rich. & Euphorbiaceae & Shrub & + & & & Not Assessed \\
\hline Acanthus montanus (Nees) T. Anders. & Acanthaceae & Forb & + & & & Least Concern \\
\hline Achyranthes aspera Linn. & Asteraceae & Forb & & & & Not Assessed \\
\hline Adenocarpus mannii Hook f. & Pailionoideae & Shrub & & & + & Not Assessed \\
\hline Adiantum philippense $\mathrm{L}$. & Adiantaceae & Fern & + & & & Not Assessed \\
\hline Adiantum sp. L. & Adiantaceae & Fern & & & + & \\
\hline Aerangis biloba (Lindl.) Schltr. & Orchidaceae & Epiphyte & + & & & Not Assessed \\
\hline Aframomum angustifolium (Sonn.) K. Schum. & Zingiberaceae & Forb & & & + & Least Concern \\
\hline Aframomит meleguetaK. Schum. & Zingiberaceae & Shrub & & & & Not Assessed \\
\hline Afzelia africana $\mathrm{Sm}$ & Caesalpinioideae & Tree & & & & Vulnerable \\
\hline Agauria salicifolia (Comm.) Hook. f. ex Oliv & Ericaceae & Tree & & & + & Not Assessed \\
\hline Ageratum conyzoides Linn. & Asteraceae & Forb & & & + & Not Assessed \\
\hline Aidia genipiflora (DC.) Dandy & Rubiaceae & Shrub & & + & & Not Assessed \\
\hline Alafia multiflora Stapf & Apocynaceae & Climber & + & & & Not Assessed \\
\hline Albizia adianthifolia (Schum.) W. F. Wight & Mimosoideae & Tree & 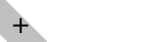 & & & Least Concern \\
\hline Albizia glaberrima (Schum. \& Thonn.) Benth. & Mimosoideae & Tree & + & + & & Not Assessed \\
\hline Albizia gummifera (J. F. Gmel.) C. A. Sm. & Mimosoideae & Tree & & + & + & Not Assessed \\
\hline Alchornea cordifolia (Schum. \& Thonn.) Müll. Arg. & Euphorbiaceae & Shrub & + & & & Not Assessed \\
\hline Alchornea laxiflora (Benth.) Pax \& K. Hoffm. & Euphorbiaceae & Shrub & & + & + & Not Assessed \\
\hline Allophylus bullatus Radlk. & Sapindaceae & Tree & & & + & Vulnerable \\
\hline Allophylus africanus P. Beauv. & Sapindaceae & Shrub & & + & & Not Assessed \\
\hline Alstonia boonei De Wild. & Apocynaceae & Tree & + & & & Not Assessed \\
\hline Amorphophallus abyssinicus (A. Rich.) N. E. & Araliaceae & Forb & + & & & Not Assessed \\
\hline Amorphophallus sp.Blume ex Decne. & & Forb & + & & & \\
\hline Anchomanes difformis (Blume) Engl. & Araceae & Forb & + & & & Not Assessed \\
\hline Andropogon tectorum Schum. \& Thonn. & Poaceae & Grass & + & + & + & Not Assessed \\
\hline Aneilema beniniense (P. Beauv.) Kunth & Commelinaceae & Forb & & + & & Not Assessed \\
\hline Aneilema lanceolatum Benth. & Comelinaceae & Forb & + & + & & Not Assessed \\
\hline Angraecum subulatum Lindl. & Orchidaceae & Epiphyte & + & & & Least Concern \\
\hline Aningeria altissima (A. Chev.) Aubrév. \& P & Sapotaceae & Tree & & & + & Not Assessed \\
\hline Annona senegalensis Pers. & Annonaceae & Tree & + & & & Not Assessed \\
\hline Anogeissus leiocarpus (DC.) Guill. \& Perr. & Combretaceae & Tree & + & & & Not Assessed \\
\hline Anthocleista djalonensis A. Chev. & Loganiaceae & Tree & & & + & Not Assessed \\
\hline Anthonotha macrophylla P. Beauv. & Caesalpinioideae & Tree & & + & & Not Assessed \\
\hline Anthonotha noldeae (Rossberg) Exell \& Hillc & Caesalpinioideae & Tree & & + & & Not Assessed \\
\hline Antiaris toxicaria Scott-Elliot ex A. Chev. & Moraceae & Tree & + & & & Not Assessed \\
\hline Antidesma membranaceum Müll. Arg. & Euphorbiaceae & Tree & + & & & Not Assessed \\
\hline Aspilia africana (Pers.) C. D. Adams & Asteraceae & Forb & & + & & Not Assessed \\
\hline Aspilia angustifolia Oliv. \& Hiern & Asteraceae & Forb & & & + & Not Assessed \\
\hline Aspilia helianthoides (Schum. \& Thonn.) Oliv. \& Hiern & Asteraceae & Shrub & & & + & Least Concern \\
\hline Asplenium dregeanum Kunze & Aspleniaceae & Fern & & & + & Not Assessed \\
\hline Asplenium preussii Hieron. & Aspleniaceae & Fern & & & + & Not Assessed \\
\hline Asplenium stuhlmannii Hieron. & Aspleniaceae & Fern & + & & & Not Assessed \\
\hline
\end{tabular}




\begin{tabular}{|c|c|c|c|c|c|c|}
\hline Asplenium theciferum (Humb. Bonpl. \& Kunth) Mett. & Aspleniaceae & Fern & & & + & Not Assessed \\
\hline Aubrevillea kerstingii (Harms) Pellegr. & Mimosoideae & Tree & + & + & & Not Assessed \\
\hline Axonopus compressus (Sw.) P. Beauv. & Poaceae & Grass & + & + & $*$ & Not Assessed \\
\hline Bambusa vulgaris Schrad. ex Wendel. & Poaceae & Shrub & + & & & Not Assessed \\
\hline Begonia macrocarpa Warb. & Begoniaceae & Forb & & & + & Not Assessed \\
\hline Beilschmiedia mannii (Meisn.) Benth. \& Hook. f. & Lauraceae & Tree & & & + & Not Assessed \\
\hline Berlinia grandiflora (Vahl) Hutch. \& Dalz. & Caesalpinioideae & Tree & + & & & Not Assessed \\
\hline Bersama abyssinica (Planch.) Verdcourt & Melianthaceae & Tree & + & & & Not Assessed \\
\hline Bidens pilosa Linn. & Asteraceae & Forb & & + & & Not Assessed \\
\hline Blighia sapida Konig & Sapindaceae & Tree & + & + & & Not Assessed \\
\hline Blighia unijugata Bak. & Sapindaceae & Tree & + & & & Not Assessed \\
\hline Bolbitis acrostichoides (Afzel. ex Sw.) Ching. & Bolbitidaceae & Fern & & & + & Not Assessed \\
\hline Bombax costatum Pellegr. \& Vuillet & Bombacaceae & Tree & + & & & Not Assessed \\
\hline Borassus aethiopum Mart. & Arecaceae & Tree & + & & & Not Assessed \\
\hline Borreria verticillata (Linn.) G. F. W. Mey. & Rubiaceae & Forb & & + & & Not Assessed \\
\hline Boswellia dalzielii Hutch. & Burseraceae & Tree & + & & & Not Assessed \\
\hline Brachiaria jubata (Fig. \& De Not.) Stapf & Poaceae & Grass & + & + & + & Not Assessed \\
\hline Brachystegia eurycoma Harms & Caesalpinioideae & Tree & + & + & & Not Assessed \\
\hline Breonadia salicina (Vahl) Hepper \& J.R.I. Wood & Rubiaceae & Tree & + & & & Not Assessed \\
\hline Bridelia atroviridis Müll. Arg. & Euphorbiaceae & Tree & + & & & Not Assessed \\
\hline Bridelia speciosa Müll. Arg. & Euphorbiaceae & Tree & + & & + & Not Assessed \\
\hline Brillantaisia nitens Lindau & Acanthaceae & Shrub & & + & & Not Assessed \\
\hline Buchnera capitata Benth & Scrophulariaceae & Forb & & + & & Not Assessed \\
\hline Bulbophyllum bequaertii De Wild. & Orchidaceae & Epiphyte & & & + & Not Assessed \\
\hline Bulbophyllum congolanum Schltr. & Orchidaceae & Epiphyte & + & & & Not Assessed \\
\hline Bulbostylis sp. Kunth & & Sedge & & & + & \\
\hline Burkea africana Hook. & Caesalpinioideae & Tree & + & & & Not Assessed \\
\hline Caesalpinia bonduc (Linn.) Roxb. & Caesalpinioideae & Shrub & & + & & Not Assessed \\
\hline Calamus deerratusMann \& Wendl & Arecaceae & Tree & + & & & Not Assessed \\
\hline Calopogonium mucunoides Desv. & Papilionoideae & Climber & & + & & Not Assessed \\
\hline Calyptrochilum christyanum (Rchb. f.) S & Orchidaceae & Epiphyte & + & & & Not Assessed \\
\hline Calyptrochilum emarginatum (Sw.) Schltr. & Orchidaceae & Epiphyte & + & & & Not Assessed \\
\hline Campylospermum flavum (Schumach. \& Thonn.) Farro & Ochnaceae & Shrub & + & + & + & Not Assessed \\
\hline Carapa proceraDC. & Asclepiadaceae & Tree & & & + & Not Assessed \\
\hline Cassipourea congoensis $\mathrm{R}$. Br. ex DC. & Rhizophoraceae & Tree & & & + & Not Assessed \\
\hline Ceiba pentandra (Linn.) Gaertn. & Bombacaceae & Tree & + & + & & Not Assessed \\
\hline Celosia trigyna Linn. & Amaranthaceae & Forb & & + & & Not Assessed \\
\hline Celtis philippensis Blanco & Ulmaceae & Shrub & + & & & Not Assessed \\
\hline Chassalia kolly (Schumach.) Hepper & Rubiaceae & Shrub & + & & & Not Assessed \\
\hline Chloris pilosa Schumach. & Poaceae & Grass & & & + & Not Assessed \\
\hline Chromolaena odorata (L.) R. M. King \& H. Ro & Asteraceae & Shrub & + & & & Not Assessed \\
\hline Chrysophullum albidum G. Don & Sapotaceae & Tree & + & & & Not Assessed \\
\hline Cissampelos mucronata A. Rich.-Diels & Menispermaceae & Climber & & + & & Not Assessed \\
\hline Cissampelos owariensis P. Beauv. ex DC. & Menispermaceae & Climber & & & + & Not Assessed \\
\hline Cissus aralioides (Welw. ex Bak.) Planch. & Vitaceae & Climber & & + & & Not Assessed \\
\hline Cissus populnea Guill. \& Perr. & Vitaceae & Climber & & + & & Not Assessed \\
\hline
\end{tabular}




\begin{tabular}{|c|c|c|c|c|c|c|}
\hline Clausena anisate (Willd.) Hook. f. ex Benth. & Rutaceae & Tree & & + & + & Not Assessed \\
\hline Cleistopholis patens (Benth.) Engl. \& Diels & Annonaceae & Tree & & + & & Not Assessed \\
\hline Clematis hirsuta Guill. \& Perr. & Ranunculaceae & Climber & & + & & Not Assessed \\
\hline Cleome ciliataSchum. \& Thonn. & Capparidaceae & Forb & & + & & Not Assessed \\
\hline Clerodendrum capitatum (Willd.) Schum. \& Thonn. & Verbenaceae & Shrub & & + & & Not Assessed \\
\hline Clerodendrum formicarum Gürke & Verbenaceae & Shrub & & & & Not Assessed \\
\hline Cnestis ferruginea DC. & Connaraceae & Shrub & & & & Not Assessed \\
\hline Cochlospermum planchonii Hook. $\mathrm{f}$. & Cochlospermaceae & Forb & & + & & Not Assessed \\
\hline Coffea brevipes Hiern & Rubiaceae & Tree & + & & & Not Assessed \\
\hline Cola gigantea A. Chev. & Sterculiaceae & Tree & + & & & Not Assessed \\
\hline Cola millenii K. Schum. & Sterculiaceae & Tree & & + & & Not Assessed \\
\hline Combretum molle R. Br. ex G. Don & Combretaceae & Tree & & & & Not Assessed \\
\hline Combretum sp. Loefl. & Combretaceae & Climber & & & & \\
\hline Commelina africana Linn. & Commelinaceae & Forb & & + & & Least Concern \\
\hline Commelina benghalensis Linn. & Commelinaceae & Forb & & & + & Not Assessed \\
\hline Commelina diffusa Burm. f. & Commelinaceae & Forb & + & & & Least Concern \\
\hline Commiphora kerstingii Engl. & Burseraceae & Tree & + & & & Not Assessed \\
\hline Connarus griffonianus Baill. & Connaraceae & Shrub & & & & Not Assessed \\
\hline Coreopsis asperata Hutch. \& Dalz. & Asteraceae & Forb & & + & & Not Assessed \\
\hline Coreopsis barteri Oliv. \& Hiern & Asteraceae & Forb & & + & & Not Assessed \\
\hline Costus afer Ker-Gawl. & Zingiberaceae & Shrub & + & & & Not Assessed \\
\hline Costus englerianus K.Schum. & Zingiberaceae & Forb & + & & & Not Assessed \\
\hline Costus spectabilis (Fenzl.) K. Schum. & Zingiberaceae & Forb & + & & + & Not Assessed \\
\hline Crassocephalum biafrae (Oliv. \& Hiern) S. Moore & Asteraceae & Forb & & + & & Not Assessed \\
\hline Crassocephalum rubens (Juss.ex Jacq.) S. Moore & & Forb & & + & & Not Assessed \\
\hline Crassocephalum sp. Moench & Asteraceae & Forb & & + & & \\
\hline Crinum jagus (Thomps.) Dandy & Liliaceae & Forb & + & + & + & Not Assessed \\
\hline Crinum zeylanicum Linn. & Liliaceae & Forb & + & & + & Not Assessed \\
\hline Crossopteryx febrifuga (Afzel. ex G. Don) Benth. & Rubiaceae & Tree & + & & & Not Assessed \\
\hline Crotalaria atrorubens Hochst. ex Benth. & Papilionoideae & Forb & & + & & Not Assessed \\
\hline Crotalaria retusa Linn. & Papilionoideae & Forb & & + & & Not Assessed \\
\hline Crotalaria sp. Linn. & Papilionoideae & Forb & & + & & \\
\hline Croton macrostachyus Hochst. ex Del. & Euphorbiaceae & Tree & & + & + & Not Assessed \\
\hline Ctenium sp. Panz. & Poaceae & Grass & + & & + & \\
\hline Culcasia scandens P. Beauv. & Araceae & Epiphyte & + & & & Least Concern \\
\hline Curculigo pilosa (Schum. \& Thonn.) Engl. & Hypoxidaceae & Forb & + & & & Not Assessed \\
\hline Cussonia barteri Seemann & Araliaceae & Tree & & + & & Not Assessed \\
\hline Cuviera nigrescens (Sc. Elliot ex Oliv.) Wernham & Rubiaceae & Shrub & & + & & Not Assessed \\
\hline Cyanotis angusta C. B. Cl. & Commelinaceae & Forb & & & + & Not Assessed \\
\hline Cyanotis longifolia Benth. & Commelinaceae & Forb & & + & & Not Assessed \\
\hline Cyathea dregei Kunze & Cyatheaceae & Fern & & & + & Not Assessed \\
\hline Cyathea manniana Hook. & Cyatheaceae & Fern & & & + & Not Assessed \\
\hline Cyperus articulates Linn. & Cyperaceae & Sedge & & + & & Least Concern \\
\hline Cyperus denudatus Linn. & Cyperaceae & Sedge & + & & & Not Assessed \\
\hline Cyperus tenuiculmis Boeck. & Cyperaceae & Sedge & + & & & Least Concern \\
\hline Dactyloctenium aegyptium (Linn.) P. Beauv. & Poaceae & Grass & + & & & Not Assessed \\
\hline
\end{tabular}




\begin{tabular}{|c|c|c|c|c|c|c|}
\hline Daniellia oliveri (Rolfe) Hutch. et Dalz. & Caesalpinioideae & Tree & + & + & + & Not Assessed \\
\hline Desmodium gangeticum (Linn.) DC. & Papilionoideae & Shrub & & + & & Not Assessed \\
\hline Desmodium repandum (Vahl) DC. & Papilionoideae & Forb & & + & & Not Assessed \\
\hline Desmodium sp. Desv. & Papilionoideae & Forb & & + & & \\
\hline Desplatsia dewevrei (De Wild. \& Th. Dur.) Burret & Tiliaceae & Tree & & & & Not Assessed \\
\hline Detarium macrocarpum Harms & Caesalpinioideae & Tree & & & & Not Assessed \\
\hline Digitaria nuda Schumach. & Poaceae & Grass & & & $*$ & Not Assessed \\
\hline Diodia sarmentosa $\mathrm{Sw}$. & Rubiaceae & Forb & & & + & Not Assessed \\
\hline Dioscorea sp. Linn. & Dioscoreaceae & Climber & & + & & \\
\hline Diospyros monbuttensis Gürke & Ebenaceae & Tree & + & & & Not Assessed \\
\hline Diplazium sammati (Kuhn) C. Christ. & Athyriaceae & Fern & & & & Not Assessed \\
\hline Dissotis bamendae Brenan \& Keay & Melastomataceae & Forb & & & + & Vulnerable \\
\hline Dissotis brazzea Long. & Melastomataceae & Shrub & & + & & Not Assessed \\
\hline Dissotis cf. decumbens (P. Beauv.) Triana & Melastomataceae & Forb & & & + & Not Assessed \\
\hline Dissotis elliotii Gilgvar. elliotii & Melastomataceae & Shrub & & + & + & Not Assessed \\
\hline Dissotis fruticose (Brenan) Brenan \& Keay & Melastomataceae & Shrub & + & + & & Not Assessed \\
\hline Dissotis rotundifolia $(\mathrm{Sm}$.$) Triana$ & Melastomataceae & Forb & + & + & & Not Assessed \\
\hline Dissotis senegambiensis (Guill. \& Perr.) Triana. & Melastomataceae & Forb & & & + & Not Assessed \\
\hline Dissotis theifolia (G. Don) Hook. f. & Melastomataceae & Shrub & & & + & Not Assessed \\
\hline Dissotis tubulosa $(\mathrm{Sm}$.$) Triana$ & Melastomataceae & Shrub & & + & + & Not Assessed \\
\hline Dombeya cf. ledermannii Engl. & Sterculiaceae & Tree & & & + & Endangered \\
\hline Doryopteris nicklesi Tard & Sinopteridaceae & Fern & + & & & Not Assessed \\
\hline Dracaena deisteliana Engl. & Agavaceae & Shrub & & & + & Not Assessed \\
\hline Dracaena mannii Bak. & Agavaceae & Tree & + & & & Not Assessed \\
\hline Dracaena phrynioides Hook. & & Tree & + & & & Not Assessed \\
\hline Drynaria volkensii Hieron. & Drynariaceae & Fern & & & + & Not Assessed \\
\hline Echinops giganteus A. Rich. & Asteraceae & Forb & & + & + & Not Assessed \\
\hline Echinops gracilis O. Hoffm. & Asteraceae & Shrub & & + & & Not Assessed \\
\hline Elaeis guineensis Jacq. & Arecaceae & Tree & + & & & Not Assessed \\
\hline Elephantopus mollis Kunth & Asteraceae & Forb & & + & & Not Assessed \\
\hline Emilia coccinea $($ Sims) G. Don & Asteraceae & Forb & & + & & Not Assessed \\
\hline Ensete gilletii (De Wild.) E. E. Cheesman & Musaceae & Forb & & & + & Not Assessed \\
\hline Entada africana Guill. \& Perr. & Mimosoideae & Tree & + & & & Not Assessed \\
\hline Entandrophragma angolense (Welw.) C. DC. & Meliaceae & Tree & + & & + & Vulnerable \\
\hline Eragrostis aspera (Jacq.) Nees & Poaceae & Grass & + & + & + & Not Assessed \\
\hline Eragrostis tenella (Linn.) P. Beauv. ex Roem & Poaceae & Grass & + & + & + & Not Assessed \\
\hline Eragrostis tenuifolia (A. Rich.) Hochst. ex Steud. & Poaceae & Grass & & & + & Not Assessed \\
\hline Eriosema glomeratum (Guill. \& Perr.) Hook. f. & Papilionoideae & Shrub & & + & & Not Assessed \\
\hline Erythrophleum suaveolens (Guill. \& Perr.) Brenan & Caesalpinioideae & Tree & + & & & Not Assessed \\
\hline Eugenia gilgi Engl. \& v. Brehm. & Myrtaceae & Tree & & + & + & Not Assessed \\
\hline Eulophia cristata (Sw.) Steud. & Orchidaceae & Forb & & & + & Not Assessed \\
\hline Eulophia horsfallii (Batem.) Summerh. & Orchidaceae & Forb & + & & & Not Assessed \\
\hline Euphorbia glomerata A. Berger. & Euphorbiaceae & Forb & + & & & Not Assessed \\
\hline Faurea speciosa Welw & Proteaceae & Tree & & & + & Not Assessed \\
\hline Ficus polita Vahl & Moraceae & Tree & & + & & Not Assessed \\
\hline Ficus sp. Linn. & Moraceae & Tree & & & + & \\
\hline
\end{tabular}




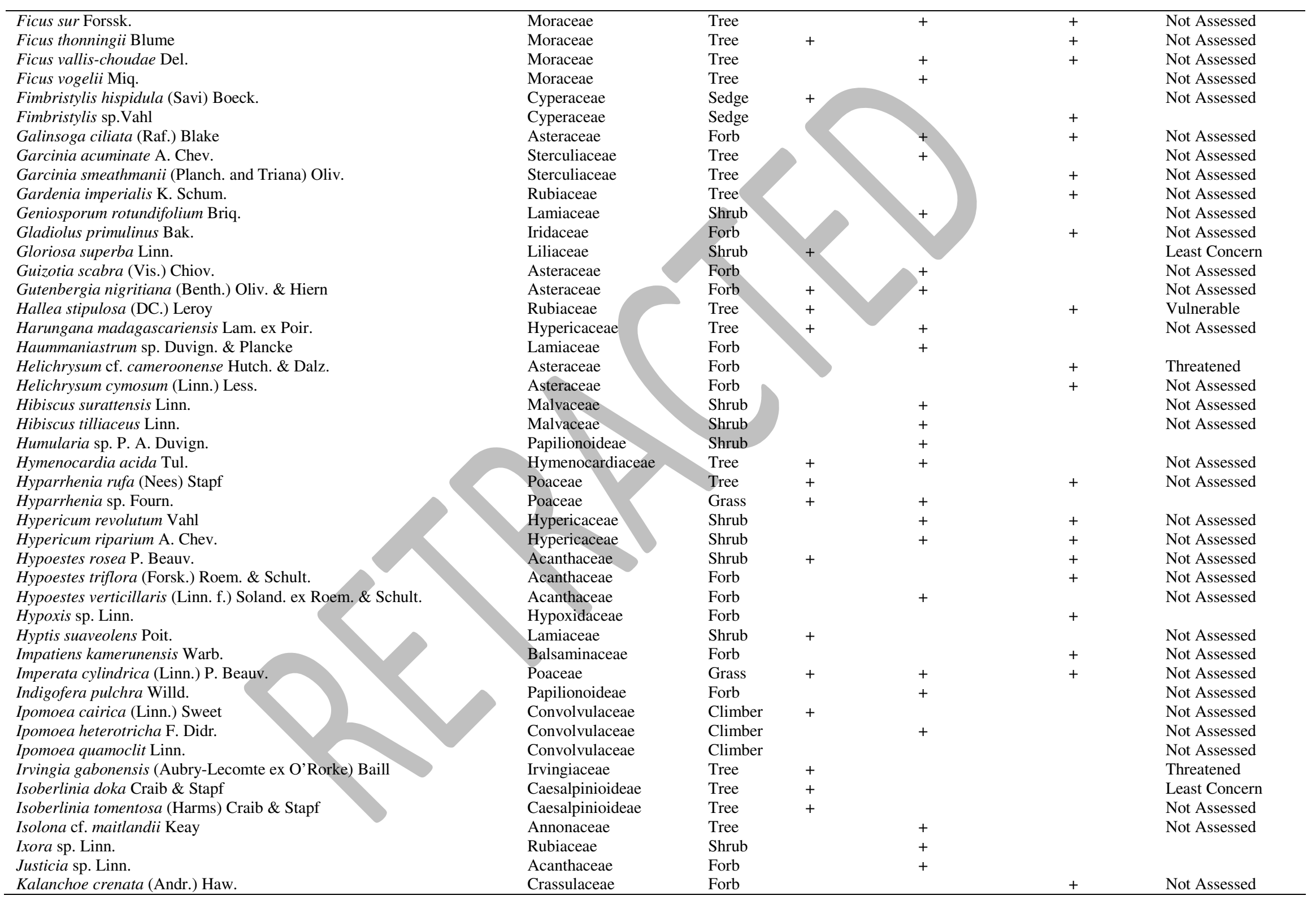




\begin{tabular}{|c|c|c|c|c|c|c|}
\hline Khaya grandifoliola C. DC. & Meliaceae & Tree & + & + & & Vulnerable \\
\hline Khaya senegalensis (Desr.) A. Juss. & Meliaceae & Tree & + & & & Vulnerable \\
\hline Kigelia africana (Lam.) Benth. & Bignoniaceae & Tree & + & & & Not Assessed \\
\hline Kyllinga bulbosa P. Beauv. & Cyperaceae & Sedge & & & + & Least Concern \\
\hline Kyllinga pumila Michx. & Cyperaceae & Sedge & 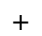 & & & Not Assessed \\
\hline Lactuca taraxacifolia (Willd.) Schum. ex Hornemann & Asteraceae & Forb & & & & Not Assessed \\
\hline Laggera aurita (Linn. f.) Benth. ex C.B. Clarke & Asteraceae & Forb & & & + & Not Assessed \\
\hline Laggera pterodonta (DC.) Sch. Bip. ex Oliv. & Asteraceae & Shrub & & + & & Not Assessed \\
\hline Lannea acida A. Rich. & Anacardiaceae & Tree & & + & & Not Assessed \\
\hline Lannea kerstingii Engl. \& K. Krause & Anacardiaceae & Tree & & + & & Not Assessed \\
\hline Lannea schimperi (Hochst. ex. A. Rich.) Engl. & Anacardiaceae & Tree & & + & & Not Assessed \\
\hline Lastreopsis subsimilis (Hook.) Tind. & Dryopteridaceae & Fern & & & + & Not Assessed \\
\hline Lecaniodiscus cupanioides Planch. ex Benth. & Sapindaceae & Tree & & + & & Not Assessed \\
\hline Lemna paucicostata Hegelm. ex Engelm. & Lemnaceae & Forb & & & & Least Concern \\
\hline Leonotis nepetifolia (Linn.) Ait. f. & Lamiaceae & Shrub & & + & & Not Assessed \\
\hline Leptaspis zeylanica Nees ex Steud. & Poaceae & Grass & + & * & & Not Assessed \\
\hline Liparis nervosa (Thunb.) Lindl. & Orchidaceae & Forb & & + & & Not Assessed \\
\hline Lippia multiflora Moldenke & Verbenaceae & Shrub & & + & & Not Assessed \\
\hline Lippia sp. Linn. & Verbenaceae & Shrub & & + & & \\
\hline Lobelia columnaris Hook. f. & Campanulaceae & Forb & & & + & Vulnerable \\
\hline Lonchocarpus sericeus (Poir.) H. B. \& K. & Papilionoideae & Tree & + & & & Not Assessed \\
\hline Lophira alata Banks ex Gaertn. f. & Ochnaceae & Tree & + & & & Vulnerable \\
\hline Lophira lanceolate Van Tiegh. ex Keay & Ochnaceae & Tree & + & + & & Not Assessed \\
\hline Loudetia simplex (Nees) C. E. Hubbard & Poaceae & Grass & & & + & Not Assessed \\
\hline Ludwigia octovalvis (Jacq.) P.H. Raven & & Forb & + & & & Least Concern \\
\hline Lycopodium cernuum Linn. & Lycopodiaceae & Fern & & & + & Not Assessed \\
\hline Lycopodium sp. Linn. & Lycopodiaceae & Fern & + & & & \\
\hline Maesa lanceolata Forsk. & Myrsinaceae & Tree & & + & + & Not Assessed \\
\hline Malacantha alnifolia (Bak.) Pierre & Sapotaceae & Tree & & + & & Not Assessed \\
\hline Mangifera indica Linn. & Anacardiaceae & Tree & + & & & Not Assessed \\
\hline Manilkara obovata (Sabine \& G. Don) J. H. Hemsl & Sapotaceae & Tree & + & & & Not Assessed \\
\hline Marantochloa purpurea (Ridl.) Milne-Redh. & Marantaceae & Forb & + & & & Not Assessed \\
\hline Margaretta rosea Oliv. & Apocynaceae & Forb & & & + & Not Assessed \\
\hline Margaritaria discoidea (Baill.) G.L. Webster & Euphorbiaceae & Tree & + & & & Not Assessed \\
\hline Mariscus flabelliformis Kunth. & Cyperaceae & Sedge & & & + & Not Assessed \\
\hline Mariscus longibracteatus Cherm. & Poaceae & Grass & + & & & Not Assessed \\
\hline Megaphrynium macrostachyum (Benth.) Milne-Redh & Marantaceae & Shrub & + & & & Not Assessed \\
\hline Microglossa angolensis Oliv. \& Hiern & Asteraceae & Shrub & & + & & Not Assessed \\
\hline Mikania cordata (Burm. f.) B.L. Robinson & Asteraceae & Climber & & + & & Not Assessed \\
\hline Milicia excelsa (Welw.) C.C. Berg & Moraceae & Tree & + & & & Threatened \\
\hline Millettia sp.Wight \& Arn. & Papilionoideae & Tree & & & + & \\
\hline Mimosa pigra Linn. & Mimosaceae & Forb & + & & & Not Assessed \\
\hline Mimusops kummel Bruce ex A. DC. & Sapotaceae & Tree & & + & & Not Assessed \\
\hline Monodora myristica (Gaertn.) Dunal & Annonaceae & Tree & & + & & Not Assessed \\
\hline Monodora tenuifolia Benth. & Annonaceae & Tree & + & & & Not Assessed \\
\hline Monotes kerstingii Gilg & Dipterocarpaceae & Tree & + & & & Not Assessed \\
\hline
\end{tabular}




\begin{tabular}{|c|c|c|c|c|c|c|}
\hline Mostuea hirsuta (T. Anders. ex Benth.) Baill. ex Bak. & Loganiaceae & Shrub & + & & & Not Assessed \\
\hline Mussaenda elegans Schum. \& Thonn. & Rubiaceae & Shrub & + & & & Not Assessed \\
\hline Mussaenda erythrophylla Schum. \& Thonn. & Rubiaceae & Shrub & + & & & Not Assessed \\
\hline Napoleona imperialis P. Beauv. & Lecythidaceae & Tree & + & & & Not Assessed \\
\hline Nephrolepis undulata (Afzelius ex Sw.) J. Sm. & Nephrolepidaceae & Fern & & + & + & Not Assessed \\
\hline Nephrolepis biserrata (Sw.) Schott. & Nephrolepidaceae & Fern & & & & Not Assessed \\
\hline Nervilia shirensis (Rolfe) Schltr. & Orchidaceae & Forb & & & & Not Assessed \\
\hline Newtonia buchananii (Baker) G. C. C. Gilbert \& Boutique & Mimosoideae & Tree & & & & Not Assessed \\
\hline Nuxia congesta $\mathrm{R}$. Br. ex Fresen. & Loganiaceae & Tree & & & + & Not Assessed \\
\hline Nymphaea lotus Linn. & Nymphaeaceae & Forb & + & & & Not Assessed \\
\hline Ocimum suave Willd. & Lamiaceae & Shrub & & + & + & Not Assessed \\
\hline Olax subscorpioidea Oliv. & Olacaceae & Shrub & & & & Not Assessed \\
\hline Opilia celtidifolia (Guill. \& Perr.) Endl. ex Walp. & Opiliaceae & Tree & & & & Not Assessed \\
\hline Oplismenus burmannii (Retz.) P. Beauv. & Poaceae & Grass & & & & Not Assessed \\
\hline Oplismenus hirtellus (Linn.) P. Beauv. & Poaceae & Grass & & & + & Not Assessed \\
\hline Oxalis corniculata Linn. & Oxalidaceae & Forb & & + & & Not Assessed \\
\hline Palisota hirsuta (Thunb.) K. Schum. & Commelinaceae & Shrub & + & & & Not Assessed \\
\hline Pandanus candelabrum P. Beauv. & Pandanaceae & Tree & + & & & Not Assessed \\
\hline Panicum maximum Jacq. & Poaceae & Grass & + & & + & Not Assessed \\
\hline Parinari excelsa Sabine & Chrysobalanac & Tree & + & & & Not Assessed \\
\hline Paspalum scrobiculatum Linn. & Poaceae & Grass & & & + & Least Concern \\
\hline Paullinia pinnata Linn. & Sapindaceae & Climber & + & + & + & Not Assessed \\
\hline Pennisetum polystachion (Linn.) Schult. & Poaceae & Grass & + & & & Not Assessed \\
\hline Pennisetum sp. Linn. Rich. & Poaceae & Grass & & & + & \\
\hline Peperomia fernandopoiana C. DC. & Piperaceae & Epiphyte & & & + & Not Assessed \\
\hline Peperomia reflexa (Linn. f.) A. Dietr. & Piperaceae & Epiphyte & & & + & Not Assessed \\
\hline Persea americana Mill. & Lauraceae & Tree & & + & + & Not Assessed \\
\hline Phaulopsis barteri (T. Anders.) Lindau & Acanthaceae & Forb & + & & & Not Assessed \\
\hline Phaulopsis falcisepala C. B. Cl. & Acanthaceae & Forb & & + & & Not Assessed \\
\hline Phillipia (Erica) mannii (Hook. f.) Beentje & Ericaceae & Shrub & & & + & Not Assessed \\
\hline Phoenix reclinata Jacq. & Arecaceae & Tree & + & & & Not Assessed \\
\hline Piliostigma thonningi (Schum.) Milne-Redhead & Caesalpinioideae & Tree & + & + & & Not Assessed \\
\hline Pittosporum viridiflorum Sims & Pittosporaceae & Tree & & & + & Not Assessed \\
\hline Platycerium alcicorne Desv. & Platyceriaceae & Fern & + & & & Not Assessed \\
\hline Platycerium angolense Welw. ex Hook & Platyceriaceae & Fern & + & & & Not Assessed \\
\hline Pleiocarpa pycnantha (K. Schum.) Stapf & Apocynaceae & Tree & & + & & Not Assessed \\
\hline Pleopeltis preussii Tardieu & Polypodiaceae & Fern & + & & & Not Assessed \\
\hline Pollia condensata C. B. Cl. & Commelinaceae & Shrub & + & & & Not Assessed \\
\hline Polyscias fulva (Hiern) Harms & Araliaceae & Tree & & + & + & Not Assessed \\
\hline Polygonum lanigerum var. africanum Meisn & Polygonaceae & Forb & + & & & Not Assessed \\
\hline Polygonum pulchrum Blume & Polygonaceae & Shrub & + & & & Least Concern \\
\hline Polysphaeria arbuscular K. Schum. & Rubiaceae & & + & + & & Not Assessed \\
\hline Polystachya dolichophylla Schltr. & Orchidaceae & Epiphyte & + & & & Not Assessed \\
\hline Polystachya sp. Hook. & Orchidaceae & Epiphyte & & & + & \\
\hline Prosopis africana (Guill. \& Pen.) Taub. & Mimosoideae & Tree & + & & & Not Assessed \\
\hline Protea madiensis Oliv. & Proteaceae & Shrub & + & + & + & Not Assessed \\
\hline
\end{tabular}




\begin{tabular}{|c|c|c|c|c|c|c|}
\hline Prunus africana (Hook. f.) Kalkman & Rosaceae & Tree & & & + & Vulnerable \\
\hline Pseudarthria hookeri Wight \& Arn. & Papilionoideae & Forb & & + & & Not Assessed \\
\hline Pseudocedrela kotschyi (Schweinf) Harms & Meliaceae & Tree & + & & & Not Assessed \\
\hline Spondianthus preussii Engl. var. preusii & Euphorbiaceae & Tree & + & & & Not Assessed \\
\hline Pseudospondias microcarpa (A. Rich.) Engl. & Anarcadiaceae & Tree & & & & Not Assessed \\
\hline Psorospermum aurantiacum Engl. & Hypericaceae & Shrub & & & + & Not Assessed \\
\hline Psorospermum febrifugum (Hook. f.) Keay \& Milne-Redhead & Hypericaceae & Shrub & & & & Not Assessed \\
\hline Psychotria chalconeura (K. Schum.) Petit & Rubiaceae & Shrub & & & + & Least Concern \\
\hline Psychotria peduncularis (Salisb.) Steyerm. & Rubiaceae & Shrub & + & + & + & Not Assessed \\
\hline Psychotria subcordata Britton & Rubiaceae & Tree & & & + & Not Assessed \\
\hline Psychotria vogeliana Benth. & Rubiaceae & Shrub & & + & & Not Assessed \\
\hline Psydrax subcordata (DC.) Bridson & Rubiaceae & Tree & & & + & Not Assessed \\
\hline Pteridium aquilinum (Linn.) Kuhn & Dennstaedtiaceae & Fern & & + & + & Not Assessed \\
\hline Pteris acanthoneura Alston. & Pteridaceae & Fern & & & & Not Assessed \\
\hline Pteris togoensis Hieron. & Pteridaceae & Fern & & & + & Not Assessed \\
\hline Pterocarpus sp.Jacq. & Papilionoideae & Tree & + & & & \\
\hline Pterygota mildbraedii Engl. & Sterculiaceae & Tree & & & + & Not Assessed \\
\hline Rangaeris rhipsalisocia (Rchb. f.) Summerh. & Orchidaceae & Epiphyte & & & & Not Assessed \\
\hline Raphia hookeri Mann \& Wendl. & Arecaceae & Tree & + & & & Not Assessed \\
\hline Raphia sudanica A. Chev. & Arecaceae & Tree & + & & & Vulnerable \\
\hline Rauvolfia caffra Sond. & Apocynaceae & Tree & & + & & Not Assessed \\
\hline Rauvolfia vomitoria Afzel. & Apocynaceae & Tree & + & & + & Not Assessed \\
\hline Rhynchelytrum repens (Willd.) C. E. Hubbard & Poaceae & Grass & + & & & Not Assessed \\
\hline Ricinodendron heudelotii (Baill.) Pierre ex Pax & Euphorbiacea & Tree & + & & & Not Assessed \\
\hline Ritchiea albersii Gilg. & Capparaceae & Tree & & & + & Not Assessed \\
\hline Rothmannia urcelliformis (Hiern) Bullock ex Robyns & Rubiaceae & Tree & & + & & Not Assessed \\
\hline Rothmannia whitfieldii (Lindl.) Dandy & Rubiaceae & Tree & + & & & Not Assessed \\
\hline Salacia sp. Linn. & Celastraceae & Tree & + & & & \\
\hline Sarcocephalus latifolius (Sm.) E. A. Bruce & Rubiaceae & Shrub & + & + & + & Not Assessed \\
\hline Satureja punctate (Benth.) Briq. & Lamiaceae & Forb & & & + & Not Assessed \\
\hline Scadoxus multiflorus (Martyn) Raf. & Amaryllidaceae & Forb & + & & & Not Assessed \\
\hline Schefflera abyssinica (Hochst. ex A. Rich.) Harms & Araliaceae & Climber & & + & + & Not Assessed \\
\hline Schizachryium sp. Nees & Poaceae & Grass & + & & & \\
\hline Scleria sp. Berg. & Poaceae & Forb & & & + & \\
\hline Scleria verrucosa Willd. & Poaceae & Grass & + & & & Not Assessed \\
\hline Selaginella myosurus (Sw.) Alston & Selaginellaceae & Fern & & + & & Not Assessed \\
\hline Selaginella sp. P. Beauv. & Selaginellaceae & Fern & + & + & & \\
\hline Senecio mannii Hook. f. & Asteraceae & Shrub & & & + & Not Assessed \\
\hline Senna obtusifolia Linn. & Caesalpinioideae & Shrub & & + & & Not Assessed \\
\hline Sesbania macrantha Welw. ex Phill. \& Hutch. & Papilionoideae & Shrub & & & + & Not Assessed \\
\hline Setaria megaphylla (Steud.) Dur. \& Schinz & Poaceae & Grass & + & & + & Not Assessed \\
\hline Setaria sp. P. Beauv. & Poaceae & Grass & & & + & \\
\hline Sida corymbosa R. E. Fries & Malvaceae & Forb & & + & & Not Assessed \\
\hline Sida linifolia Juss. ex Cav. & Malvaceae & Shrub & & + & & Not Assessed \\
\hline Siphonochilus aethiopicus (Schweinf.) B.L. Burtt & Zingiberaceae & Forb & + & & & Not Assessed \\
\hline Siphonochilus nigericus (Hutch. ex Hepper) B.L. Burtt & Zingiberaceae & Forb & + & & & Not Assessed \\
\hline
\end{tabular}




\begin{tabular}{|c|c|c|c|c|c|c|}
\hline Smilax kraussiana Meisn. & Smilacaceae & Climber & + & & + & Not Assessed \\
\hline Solanum aculeastrum Dunal & Solanaceae & Shrub & & & + & Not Assessed \\
\hline Solanum dasyphyllum Schum. \& Thonn. & Solanaceae & Shrub & & + & & Not Assessed \\
\hline Solanum sp. Linn. & Solanaceae & Shrub & + & & & \\
\hline Solanum wrightii Benth. & Solanaceae & Shrub & & & + & Not Assessed \\
\hline Solenostemon monostachyus (A. Chev.) Brenan & Lamiaceae & Forb & & & & Not Assessed \\
\hline Sporobolus africanus (Poir.) Robyns \& Tournay & Poaceae & Grass & & & + & Not Assessed \\
\hline Sterculia rhinopetala K. Schum. & Sterculaiceae & Tree & + & & & Not Assessed \\
\hline Sterculia sp. Linn. & Sterculiaceae & Tree & + & & & \\
\hline Sterculia tragacantha Lindl. & Sterculiaceae & Tree & & + & & Not Assessed \\
\hline Stomatanthes africanus (Oliv. \& Hiern) R.M. King \& H. Rob. & Asteraceae & Forb & + & + & & Not Assessed \\
\hline Striga aspera (Willd.) Benth. & Scrophulariaceae & Forb & & & & Not Assessed \\
\hline Strombosia scheffleri Engl. & Olacaceae & Tree & & & + & Not Assessed \\
\hline Stylochiton barteri N. E. Br. & Araceae & Forb & & & & Not Assessed \\
\hline Symphonia globulifera Linn. f. & Clusiaceae & Tree & + & + & + & Not Assessed \\
\hline Syzygium guineense (Wild.) DC. & Myrtaceae & Tree & + & + & & Not Assessed \\
\hline Syzygium guineense (Wild.) DC. subsp. bamendae & Myrtaceae & Tree & & & + & Not Assessed \\
\hline Syzygium guineense (Wild.) DC. subsp. guineense & Myrtaceae & Tree & & & + & Not Assessed \\
\hline Tabernaemontana contorta Stapf & Apocynaceae & Tree & & + & & Not Assessed \\
\hline Tabernaemontana pachysiphon Stapf & Apocynaceae & Tree & + & & & Not Assessed \\
\hline Tagetes erecta Linn. & Asteraceae & Forb & & + & & Not Assessed \\
\hline Tapinanthus bangwensis (Engl. \& K. Krause) Danser & Loranthaceae & Epiphyte & & & + & Not Assessed \\
\hline Tephrosia vogelii Hook. f. & Papilionoideae & Shrub & & + & & Not Assessed \\
\hline Terminalia avicennioides Guill. \& Perr. & Combretaceae & Tree & + & & & Not Assessed \\
\hline Terminalia glaucescens Planch. ex Benth. & Combretaceae & Tree & + & + & & Not Assessed \\
\hline Terminalia laxiflora Engl. & Combretaceae & Tree & & + & & Not Assessed \\
\hline Terminalia schimperiana Hochst. & Combretaceae & Tree & + & & & Not Assessed \\
\hline Terminalia superba Engl. \& Diels & Combretaceae & Tree & + & & & Not Assessed \\
\hline Tetrapleura tetraptera (Schum. \& Thonn.) Taub. & Mimosoideae & Tree & + & & & Not Assessed \\
\hline Thalia welwitschii Ridl. & Marantaceae & Shrub & + & & & Not Assessed \\
\hline Thonningia sanguinea Vahl. & Balanophoraceae & Epiphyte & + & & & Not Assessed \\
\hline Tithonia diversifolia (Hemsl.) A.Gray & Asteraceae & Shrub & & + & & Not Assessed \\
\hline Trema orientalis (L.) Blume & Ulmaceae & Tree & & + & + & Not Assessed \\
\hline Trichilia preuriana A. Juss & Meliaceae & Tree & + & & & Not Assessed \\
\hline Trichomanes sp. Linn. & Hymenophyllaceae & Fern & & & + & \\
\hline Trilepisium madagascariense DC. & Moraceae & Tree & + & & & Not Assessed \\
\hline Triumfetta dubia De Wild. & Tiliaceae & Shrub & & + & & Not Assessed \\
\hline Uapaca heudelotii Baill & Euphorbiaceae & Tree & + & & & Not Assessed \\
\hline Uapaca togoensis $\mathrm{Pax}$ & Euphorbiaceae & Tree & + & + & + & Not Assessed \\
\hline Urelytrum sp. Hack. & Poaceae & Grass & + & & & \\
\hline Urena lobata Linn. & Malvaceae & Shrub & + & & & Not Assessed \\
\hline Urginea altissima (Linn. f.) Bak. & Hyacinthaceae & Forb & & & + & Not Assessed \\
\hline Vernonia ambigua Kotschy \& Peyr. & Asteraceae & Shrub & & + & & Not Assessed \\
\hline Vernonia calvoana Hook. f. & Asteraceae & Shrub & & + & & Not Assessed \\
\hline Vernonia glaberrima Welw. ex O. Hoffm. & Asteraceae & Shrub & & + & & Not Assessed \\
\hline Vernonia glabra (Steetz) Vatke & Asteraceae & Forb & & + & & Not Assessed \\
\hline
\end{tabular}




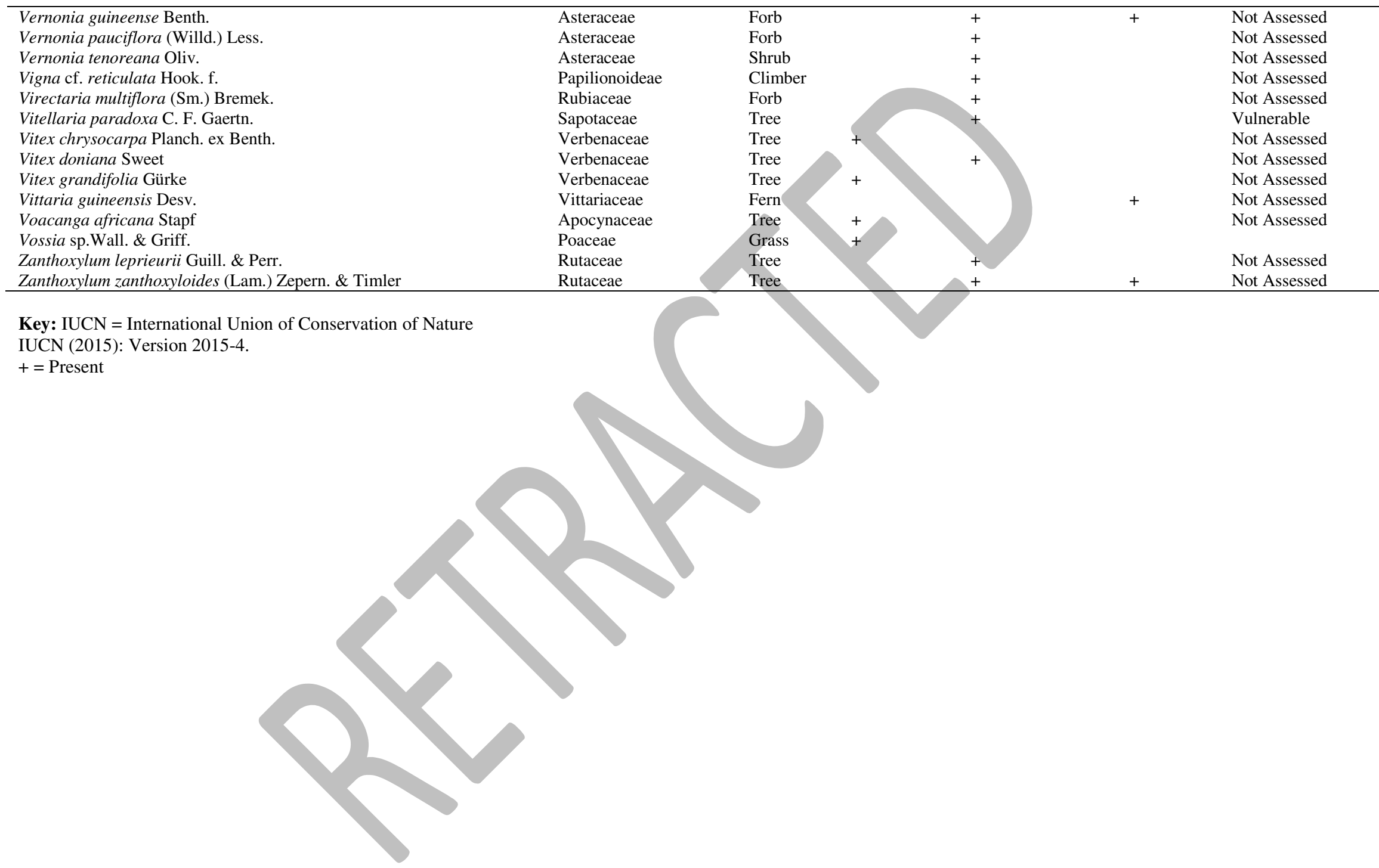




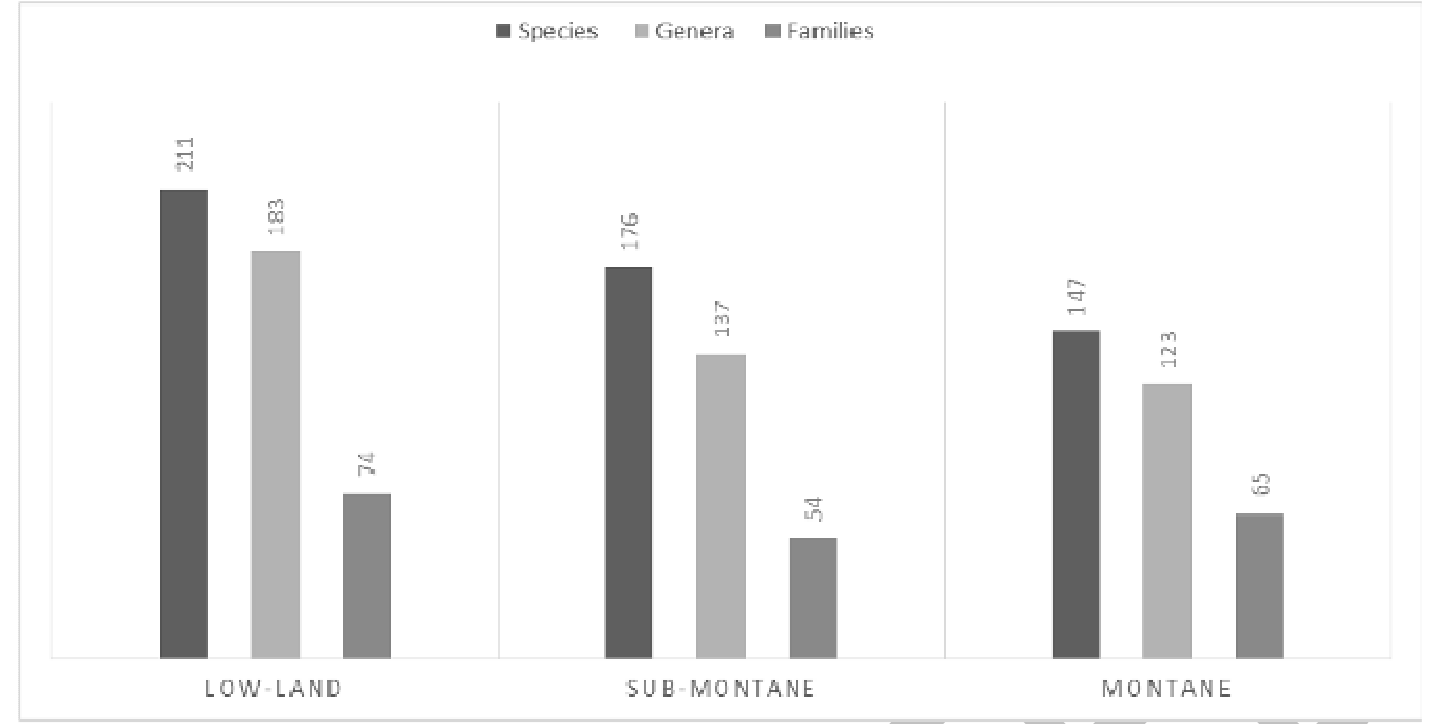

Figure 4: Summary of species composition along the altitudinal regions

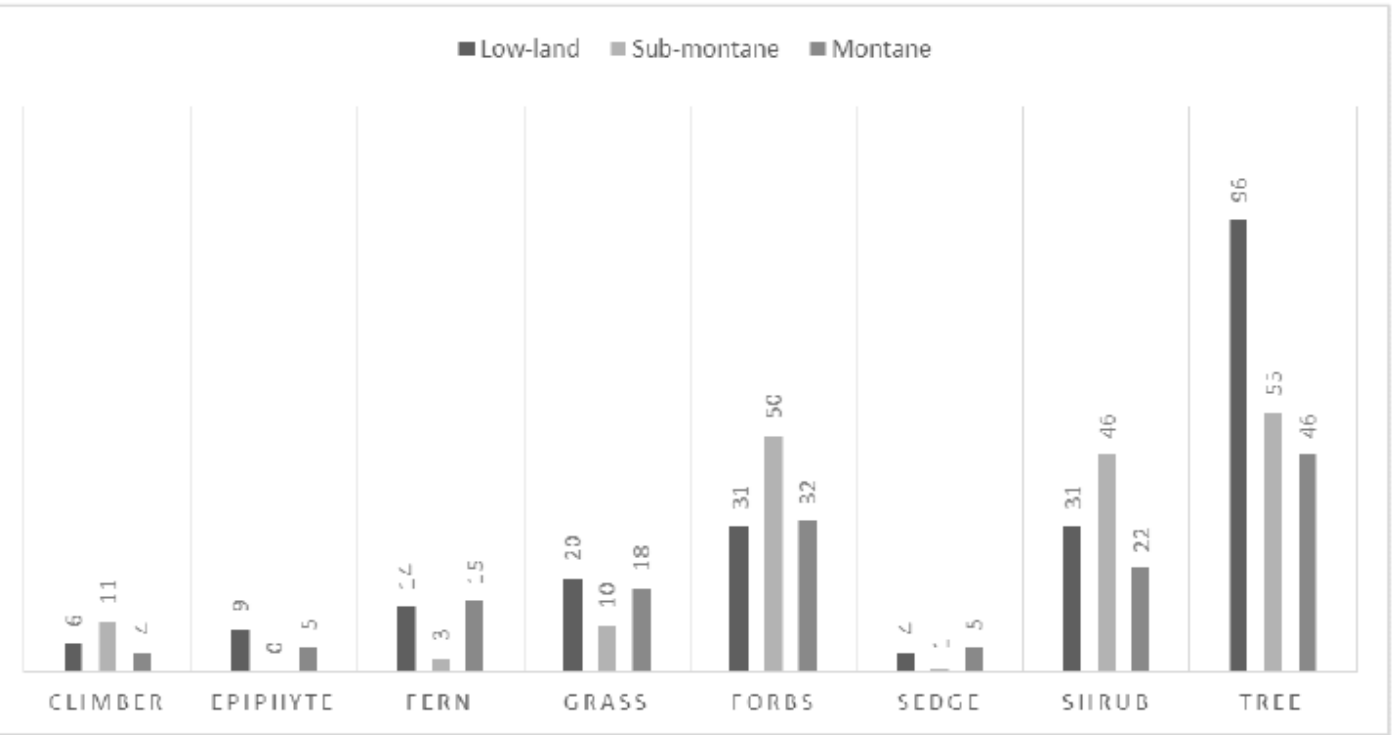

Figure 5: Summary of species habits distribution across the studied site

\section{Discussion}

Several authors have published flora and checklists of Nigerian plants; they include Hutchinson and Dalziel (1954, 1958, 1963, 1968, 1972) on angiosperms, gymnosperms and pteridophytes, Alston (1959) on ferns, Keay et al. (1964) on trees, Lowe and Stanfield (1974) on sedges, and Akobundu and Agyakwa (1998) on weeds, as well as Ayodele and Yang (2012) on vascular plants. Akinsoji
(1994, 1996), Akinsoji (2003), Akinsoji et al. (2003) and Akinsoji (2005) are the only works on the vegetation of GGNP known to the authors. Good as these works were, they cannot be said to have appropriately documented the flora of the GGNP since they are all vegetation specific. As a result of this there is the urgent need to formally document extensively the various plants of the vegetationally complex National Park 
along its geomorphic gradient as a sectoral project. This is because the park remains one of the two least disturbed and the largest in the country with peculiar combination of several vegetation types mimicking the complex Nigerian vegetation.

\section{Vegetation Types}

Lowland rainforest: A lowland tropical rainforest type comprises the following vertical tiers: emergent canopy, base canopy, middle tier and forest understory. Lowland forest was only present in the lowland region, particularly along the riparian areas of the Park. Gumnior and Sommer (2012) using remote sensing reported similar findings. Emergent trees may attain heights of 35 to $50 \mathrm{~m}$, and sometimes even higher; these species appear to poke through an otherwise fairly even canopy top, whose height is typically about 25 to $30 \mathrm{~m}$. Emergent trees in the region include Khaya grandifolia, Ceiba pentandra, Milicia excelsa and Terminalia superba while the base canopy consists of Albizia spp., Erythrophleum suaveolens and Ficus spp. Mid tier plants consist of intermediate height trees, tall shrubs and epiphytes that do not require the light intensity of the canopy trees such as Celtis philippensis and Olax subscorpioidea. The forest floor receives relatively little sunlight, and thus is rarely choked with vegetation; nevertheless, one finds here a myriad of low growing shrubs, ground trailing lianas, ferns, lichens and mosses. Expectedly, this forest support a plethora of terrestrial avafauna,

arthropoda, amphibians and reptiles as observed during this study.

Derived savanna: This zone is characterized by mosaic of vegetation because it shares ecological characteristics from both the lowland and montane vegetation. This was observed along Selbe, Tonga and Yakuba routes with altitude between 1000 and $1500 \mathrm{~m}$ asl. The derived savanna resulted from farming and grazing pressure. In this vegetation type, the flora is now characterized by grasses such as Andropogon tectorum, Hyparrhenia spp., Urelytrum sp. and Imperata cylindrica. Associated herbaceous species include Scadoxus multiflorus and Stomatanthes africanus while woody species include Sarcocephalus latifolius, Piliostigma thonningi and Combretum sp.

Montane vegetation: During this study, the highest elevation was observed at Chabbal Hendu with altitude up to $1900 \mathrm{~m}$ asl which is characteristic of this montane region. However, according to Akinsoji (1996), the highest point on the Gotel Mountains (2419 m asl) is Gangrewal, located in the southeastern fringe of the park. The floristic composition differs from one point to another along the altitude because of slope, fire, grazing etc. leading to the formation of mosaic vegetation. The observed mosaic vegetation is made up of montane forest and grassland.

Chabbal Hendu is located on the altitude range between 1800 and $2000 \mathrm{~m}$ asl with a conspicuous influence of altitude on vegetation. The forests do not show any emergents but the canopy layer is discernible although it does not form a complete cover. Canopy trees include Albizia gummifera, Symphonia globulifera, Croton monostachyus, Syzygium guineense subsp. bamendae, Prunus africana, Ficus spp., Entandrophragma angolense, Strombosia scheffleri, Garcinia smeathmanni, 
Carapa procera, Bridelia speciosa, Campylospermum flavum, Clausena anisata, Trema orientalis and Ritchiea albersii. Some specific characteristics such as the bark thickness that ranges between 0.2 and $1.5 \mathrm{~cm}$, and buttresses as seen on Syzygium and stilt roots as observed on Anthocleista were observed on the trees in this region. Also, the barks are heavily coated with lichen patches and 'pads' of epiphytic bryophytes and pteridophytes such as Asplenium dregeanum, Asplenium preussii, Asplenium theciferum, and Nephrolepis undulata.

The shrub layer is dominated by Psychotria peduncularis, Chassalia kolly, Dracaena deisteliana, Desmodium rependum and Ocimum suave while the gaps are dominated by herbaceous species such as Commelina benghalensis and Ageratum conyzoides, and juveniles of trees such as Dombeya cf. ledermannii, Milletia sp., and Clausena anisata. Lianes identified are Smilax kraussiana and Paullinia pinnata.

The grassland has been subjected to annual fire and grazing. The effect of both fire and grazing has prevented the forest extension, changed the floristic composition and allowed the development and expansion of fire resistant forest edge species like Hypericum revolutum which form thickets at the forest edge as observed at Chabbal Hendu. There is woodland vegetation along the mountain sides at slightly lower altitudes between Selbe and Chabbal Hendu. Common grasses identified include Chloris pilosa, Eragrostis spp., $\quad$ Paspalum scrobiculatum, Sporobolus africanus and Pennisetum sp. Trees include Entada africana, Combretum molle, Lophira lanceolata and Annona seneglensis while scattered shrubs are Sarcocephalus latifolius, Protea madiensis, Gardenia imperalis and Solanum aculeastrum.

Southern Guinea Savanna: Apart from the montane and lowland forest in the southern parts of the park, the rest of the vegetation is savanna. The Southern Guinea Savanna occurs in the lowland and sub-montane regions. It is characterized by the presence of grasses which are subjected to annual fires while the trees, shrubs and forbs were considerably scattered along the terrain. Dominant woody species are Lophira lanceolata, Daniellia oliveri, Afzelia africana, Crossopteryx febrifuga, Piliostigma thonningii, Entada africana, Prosopis africana, Annona senegalensis, Combretum spp., and Terminalia schimperiana. Also, characteristic dominant grasses include Andropogon tectorum, Hyparrhenia rufa, Pennisetum polystachion, Ctenium sp. and Schizachryum sp.

On the hilly side, some species such as Uapaca togoensis, Hymenocardia acida, Piliostigma thonningi and Bridelia atroviridis were found to occur in pure stands with their stems and branches covered by epiphytic species of lichens, mosses, ferns (Platycerium spp.) and some orchids such as Aerangis biloba, Angraecum subulatum, Bulbophyllum congolanum, Calyptrochilum christyanum, Calyptrochilum emarginatum, Eulophia horsfallii, Polystachya dolichophylla, and Rangaeris rhipsalisocia. Single stands of Anogeissus leiocarpus with intermittent occurrence of Borassus aethiopium were observed on moister soils along the streams in the valley. 
Other species in this sub-ecosystem include Khaya senegalensis, Alchornea cordifolia, Mussaenda elegans, Panicum maximum, Setaria megaphylla, Raphia sudanica, Phoenix reclinata, Breonadia salicina, Brachystegia eurycoma, Berlinia grandiflora, Erythrophleum suaveolens, Polysphaeria arbuscula, Vitex chrysocarpa, Uapaca heudelotii and Pandanus candelabrum. Other noticeable geophytic forbs include Costus spectabilis (the Nigeria National flower), Crinum zeylanicum, Crinum jagus, Amorphophallus sp., Amorphophalus abyssinicus, Scadoxus multiflorus, Siphonochilus aethiopicus, Curculigo pilosa, Gloriosa superba, and a shrub Cochlospermum planchonii which flowers immediately after fires before leaves are produced.

\section{Diversity}

The 426 plant species identified in this region accounts for one-fifteenth of the total vascular plants diversity in Nigeria as reported from the compilations of Ayodele and Yang (2012). Diversity in the region was further enriched by its proximity to Cameroon and this led to the occurrence of certain East African flora which were not reported by Hutchinson and Dalziel (1954) in West Tropical Africa and Ayodele and Yang (2012) in Nigeria. The new additions are; Anthonotha noldeae (Rossberg) Exell \& Hill, Psychotria subcordata Britton, Pterygota mildbraedii Engl., Tagetes erecta Linn., and Tithonia diversifolia (Hemsl.) A. Gray.

\section{Conservation}

IUCN protected area management categories classify protected areas according to their management objectives. GGNP is a category II protected area because it is a National
Park. Category II protected areas are large natural or near natural areas set aside to protect large-scale ecological processes, along with the complement of species and ecosystems characteristic of the area, which also provide a foundation for environmentally and culturally compatible spiritual, scientific, educational, recreational and visitor opportunities (IUCN, 2014).

Several of the species in this report have not been assessed by IUCN till date. Nonetheless, twelve vulnerable species were identified out of which ten are trees and two are forbs. Lophira alata, Khaya senegalensis, Khaya grandifoliola, Entandrophragma angolense, Allophylus bullatus, Hallea stipulosa, Prunus africana, and Afzelia africana are vulnerable trees mainly felled for timber in Africa. Vitellaria paradoxa is harvested for its shea butter while Raphia sudanica is over-exploited for palm wine in Northern Nigeria (Burkill, 1985). Strict policies should be developed and enforced in GGNP and Nigeria as a whole to prevent felling and over-and unsustainable-exploitation of these species.

Vulnerable herbaceous species are Lobelia columnaris and Dissotis bamendae. Lobelia columnaris is not known for any use, however, its reduction in population may be as a result of the reduction of its habitat as reported by Cheek et al. (2000). They reported that the plant is usually threatened by fires from grassland moving into forest edges; forest loss due to timber extraction and clearance for agricultural land. Similarly, Dissotis bamendae has no known use except for ornamental purposes but its vulnerability is attributed to the conversion of natural habitat to 
farmland, for grazing and for cultivation; increasing frequency of fires may also pose a threat to this species. These forbs can be conserved by reducing forest loss to agriculture or creating biodiversity offsets when such lands are to be occupied.

Three species were threatened from the list: Irvingia gabonensis, Helichrysum cf. cameroonense, and Milicia excelsa. According to IUCN (2015), Irvingia gabonensis population have declined due to logging operations, expansion of human settlements and poor natural regeneration.Other reasons may be over-exploitation of the plant for its fruits. Milicia excelsa is threatened because it suffers from heavy exploitation as source of timber, even for exports. The plant is also prone to gall attacks, especially in plantations, and its seed looses viability quickly (IUCN, 2015). Dombeya cf. ledermannii was the only endangered species in the location as at the time of this study. IUCN (2015) discloses that this endangerment is as a result of vegetation clearance for agriculture and over-exploitation of the plant for its bast fibres.

\section{GIS Analysis}

The contribution of remote sensing to ecology has been intensely documented. However, remote sensing for GGNP will be better explained using elevation models because of the characteristic geomorphic gradient of the park. Volarik (2010) explained that Digital Elevation Model (DEM) contains information both on altitude and topography and considered it to be a useful tool for transferring the knowledge of vegetation tiers from easily classifiable sites to the sites that are not easily classifiable. This indicated the dominance of this method over spatial characterization used by Gumnior and Sommer (2012) and Mubi and Tukur (2012). Volarik (2010) further stressed that DEM determines the spatial resolution of all derived maps, such as a map of slope, aspect, and curvatures. DEM is considered to be the main prerequisite map for spatial modelling in ecology and has been used as a source of variables in numerous vegetation studies such as Davis and Goetz (1990), Del Barrio et al. (1997), Gottfried et al. (1998) and Guisan et al. (1998).

Result from the ground truthing data for this study is similar to that of the DEM except in the sub-montane classification between 933 and $1467 \mathrm{~m}$. In this classification, w hile the ground truthing data categorized elevations 1500 $\mathrm{m}$ and $1588 \mathrm{~m}$ as sub-montane, the DEM categorized the elevations under montane. This is one of the advantages of using DEM in vegetation analysis of mountainous regions. Normalised Difference Vegetation Index (NDVI) can be calculated from satellite imagery and is generally recognised as a reliable index of ground vegetation cover (Hess et al., 1996). Therefore, the higher indices reflected by imagery from 1991 indicated higher vegetation cover than 2013. This difference is due to natural and anthropogenic factors, the combination of which determines the species composition of vegetation (Bakker and ter Heerdt, 2005).

\section{Conclusion}

Effective and efficient conservation with adequate management of species habitats in national parks can be achieved through proper eco-taxonomic studies, reducing the effect of anthropogenic and natural factors in reserved areas to 
minimum threshold. Ecological studies alongside GIS analysis have revealed the rate of degradation within the studied area. To the best knowledge of the authors, this is lacking in previous works on the GGNP. Therefore, we recommend that a detailed ecological studies should be carried out within GGNP and other reserved areas in Nigeria to know their biodiversity status which will later serve as a guide to formulate a better policy and law to protect our national heritage.

\section{References}

Akinsoji, A. (1994). Vegetation of Gashaka Gumti National Park, Nigeria: A preliminary survey. Unpublished Report for NCF, WWF-UK Conservation Project.

Akinsoji, A. (1996). Vegetation types and ethnobotanical studies of Gashaka Gumti National Park-Nigeria. Unpublished Report submitted to: Nigeria Conservation Foundation (NCF-Gashaka Gumti Project), NPS and WWF-UK.

Akinsoji, A. (2003). Vegetation studies of Gashaka Gumti National Park, Nigeria, I. Ethnobotany. The Nigerian Field, 68: 124-14.

Akinsoji, A. Ayanbamiji, T. and Durugbo, E.U. (2003). Comparative phytosociological analyses in the Gashaka-Gumti National Park, Nigeria using Point-Centered Quarter and Quadrat methods. Nigerian Journal of Botany, 16: 133-143

Akinsoji, A. (2005). A survey of Montane epiphytes in GashakaGumti National Park, Nigeria. Nigerian Journal of Botany, 18: 3545
Akobundu, I.O. and Agyakwa, C.W. (1998). A handbook of West African weeds. I.I.T.A., Ibadan.

Alston, A. H. G. (1959). The Ferns and Fern-allies of West Tropical AfricaCrown Agents for Overseas Governments and Administrations, London.

Angiosperm Phylogeny Group III (2009). An update of the Angiosperm Phylogeny Group classification for the orders and families of flowering plants: APG III. Botanical Journal of the Linnean Society, 161: 105121.

Ayodele A.E. and Yang, Y. (2012). Diversity and Distribution of Vascular Plants in Nigeria. Qingdao Publishing House, China. $350 \mathrm{pp}$.

Bakker J.P. and ter Heerdt G.N. (2005). Organic grassland farming in the Netherlands: a case study of effects on vegetation dynamics. Basic Applied Ecology, 6:205-214

Burkill (1985).The useful plants of West Tropical Africa, Royal Botanical Garden, ed. 2, 1: 957pp.

Chapman, H., Olson, S., Sessions, L. and Trumm, D. (2004). An assessment of changes in the montane forests ofTaraba State, Nigeria, over the past 30 years. Oryx, 38(3): 1-9.

Cheek, M., Onana, J.M. and Pollard, B.J. (2000). The plants of Mount Oku and the Ijim Ridge, Cameroon. Royal Botanic Gardens, Kew. 215 pp.

Davis, F.W. and Goetz, S. (1990). Modeling Vegetation Pattern Using Digital Terrain Data. Landscape Ecology, 4(1): 69-80.

Del Barrio, G., Alvera, B., Puigdefabregas, J. and Diez, C. 
Evaluation and Flora Diversity of Gashaka Gumti National Park-1.................AKINSOJI et al.

(1997). Response of High Mountain Landscape to Topographic Variables: Central Pyrenees. Landscape Ecology, 12: 95-115.

Dunn, A. (1995). Gashaka Gumti, Nigeria - From Game Reserve to National Park. RDFN Paper 18d, Winter 1994 B Spring 1995 1-3.

Gottfried, M., Pauli, H. and Grabherr, G. (1998). Prediction of Vegetation Patterns at the Limits of Plant Life: A New View of the Alpine-Nival Ecotone. Arctic and Alpine Research, 30: 207-221.

Guisan, A., Theurillat, J. P. and Kienast, F. (1998). Predicting the Potential Distribution of Plant Species in an Alpine Environment. Journal of Vegetation Science, 9: 65-74.

Gumnior, M. and Sommer, V. (2012). Multi-Scale, Multi-Temporal Vegetation Mapping and Assessment of Ecosystem Degradation at Gashaka Gumti National Park (Nigeria).Research Journal of Environmental and Earth Sciences, 4(4): 397-412.

Hess, T., Stephens, W. and Thomas, G. (1996). Modelling NDVI from decadal rainfall data in the North East Arid Zone of Nigeria. Journal of Environmental Management 48(3): 249-261.

Hutchinson, J.and Dalziel, J.M. (1954). Flora of West Tropical Africa, vol 1 part 1. London: Crown Agents. 295 pp.

Hutchinson, J. and Dalziel, J.M. (1958). Flora of West Tropical Africa, vol 1 part 2. London: Crown Agents. 532 pp.

Hutchinson, J. and Dalziel, J.M. (1963). Flora of West TropicalAfrica, vol 2. London: Crown Agents. 544 pp.
Hutchinson, J. and Dalziel, J.M. (1968). Flora of West Tropical Africa, vol 3 part 1. London: Crown Agents. 276 $\mathrm{pp}$

Hutchinson, J. and Dalziel, J. M. (1972). Flora of West Tropical Africa, vol 3 part 2. London: Crown Agents. 300 pp.

IUCN (2014). Protected Areas Category II. Retrieved from http://www.iucn.org/about/work/pr ogrammes/gpap_home/gpap_qualit y/gpap_pacategories/gpap_pacatego ry 21

IUCN (2015). IUCN Red List of Threatened Species. Version 2015.4. Www.iucnredlist.org. Downloaded between 1February 2016 and 1 March 2016.

Keay, R.W.J. (1959). An Outline of Nigerian Vegetation (3rd Edition), Government Printer, Lagos. 485 pp.

Keay, R.W.J., Onochie, C.F.A. and Stanfield, D. P. (1964). Nigerian Trees. Federal Department of Forest Research, Ibadan, Nigeria.

Ladipo, D. (2010). The state of Nigeria's forests. IITA Research for DevelopmentReviews (specials) 4. Retrieved from http://r4dreview.org/2010/04/thestate-of-nigeriasforests/

Lowe, J. and Stanfield, D.P. (1974). Sedges (family Cyperaceae). Ibadan University Press, Nigeria. 144 pp.

Mubi, A.M. (2010a). Remote SensingGISsupported land cover analysis of Gashaka-Gumti National Park, Nigeria. FUTY Journalof the Environment, 5(1): 15-28.

Mubi, A.M. (2010b). Assessment of TreeSpecies Distribution in Gashaka-GumtiNational Park, Nigeria: A Regression BasedModel 
Approach. The Zaria Geographer, 18: 11-13.

Mubi, A.M. and Tukur, A.L. (2012). Species density and diversity along geomorphic gradient in Gashaka Gumti National Park (GGNP), Nigeria. Ethiopian Journal of Environmental Studies and Management, 5(4):513-520.

Oates, J. F., Bergl, R.A. and Linder, J.M. (2004). Africa's Gulf of Guinea Forests: Biodiversity Patterns and Conservation Priorities. Advances in Applied Biodiversity Science 6. Conservation Internationa, Washington DC. 94 pp.
Radford, A.E., Dickison, W.C., Massey, J.R. and Bell, C.R. (1974). Vascular Plant Systematics. Harper and Row, New York. 891 pp.

Sommer, V. and Ross, C. (2011). Exploring and Protecting West Africa's Primates. The Gashaka Primate Project in Context. In: Sommer, V. and Ross, C. (Eds.), Primates of Gashaka. Socioecology and Conservation in Nigeria's Biodiversity Hotspot. Springer, New York. 1-37.

Volarik, D. (2010). Application of Digital Elevation Model for Mapping Vegetation Tiers. Journal of Forest Science 56(3): 112-120. 